\title{
A VERSATILIDADE DO ELETRODO DE GOTA PENDENTE DE MERCÚRIO EM QUÍMICA ANALÍTICA - UMA REVISÃO SOBRE RECENTES APLICAÇÕES
}

\author{
Chalder Nogueira Nunesa, Vanessa Egéa dos Anjos ${ }^{\mathrm{b}}$ e Sueli Pércio Quináiaa,* \\ a'Departamento de Química, Universidade Estadual do Centro-Oeste, 85040-080 Guarapuava - PR, Brasil

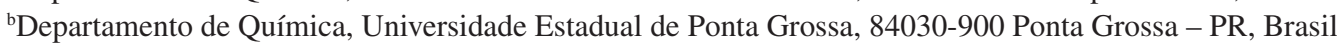

Recebido em 30/05/2017; aceito em 20/09/2017; publicado na web em 13/11/2017

\begin{abstract}
THE VERSATILITY OF THE HANGING MERCURY DROP ELECTRODE IN ANALYTICAL CHEMISTRY - A REVIEW ABOUT RECENT APPLICATIONS. Voltammetry using mercury electrode as working electrode has been employed in the determination of organic and inorganic compounds in several samples. A review on the applications of this analytical tool is present, covering the period of the last ten years. The emphasis of this work is directed to the hanging mercury drop electrode (HMDE), its advantages, disadvantages and applications. Voltammetric measurements are easily carried out, and present low cost. The review discusses that the use of mercury in the measurements can be a limitation due the toxicity of the element. However, nowadays the necessary of volume of $\mathrm{Hg}$ in the measurements is very small, and it can be reused. Thus, the correct disposal of the small amount of waste generated can be minimize environmental risks and human health, according to the principles of Green Chemistry.
\end{abstract}

Keywords: applications; mercury electrode; review; voltammetry.

\section{INTRODUÇÃO}

Técnicas eletroquímicas têm sido usadas por muitos anos devido as suas características vantajosas, tais como elevada frequência analítica, simplicidade, baixo custo, capacidade de detecção em níveis de concentração baixa, resposta dependente das formas químicas de um mesmo elemento, análise de misturas, entre outras. Os primeiros estudos eletroquímicos iniciaram no século XVIII na Itália e evoluíram até os métodos atuais, envolvendo diversos tipos de eletrodos indicadores e de trabalho, como eletrodos sólidos, de mercúrio, ultramicroeletrodos, etc. ${ }^{1-4}$ Cada tipo de eletrodo desenvolvido possui vantagens e desvantagens intrínsecas. Eletrodos de trabalho sólidos, por exemplo, apresentam como vantagem a possibilidade de serem modificados, aumentando assim sua seletividade, além de possuírem diversas formas e tamanhos que ampliam sua aplicabilidade. Entretanto, processos de limpeza e polimento são comumente necessários devido ao envenenamento da superfície eletrolítica, que podem comprometer a reprodutibilidade de sua área superficial. O eletrodo de gota pendente de mercúrio, do inglês hanging mercury drop electrode (HMDE), por sua vez, por ser renovável apresenta excelente reprodutibilidade, uma superfície extremamente lisa e uniforme a cada gota. Além disso, um elevado sobrepotencial à redução de hidrogênio, uma ampla faixa negativa de potencial (de 0 a $-2 \mathrm{~V}$ dependendo do eletrólito suporte) assim como a superfície reprodutível a cada gota, são vantagens deste eletrodo. ${ }^{5}$ Entretanto, o HMDE apresenta baixa resistência mecânica, limitando suas aplicações, bem como relativa seletividade para compostos orgânicos, pode sofrer com interferências causadas por matéria orgânica dissolvida em amostras ambientais, estreita faixa de trabalho na região anódica, etc. Além disso, muitas discussões vêm sendo realizadas com relação à toxicidade do mercúrio e a necessidade de buscar materiais alternativos para o desenvolvimento de outros eletrodos de trabalho mais condizentes com os aspectos de Química Verde. Contudo, é importante salientar que após ser usado, o mercúrio $(\mathrm{Hg})$ pode ser purificado com elevado grau de pureza $(99,9999 \%)$ por meio de destilação, seguida por lavagem com ácido nítrico diluído e reutilizado. ${ }^{6}$ Além disso, a quantidade de resíduos

*e-mail: spquinaia@unicentro.br de $\mathrm{Hg}$ gerados através dos métodos mais atuais é bastante reduzido, minimizando os aspectos negativos do $\mathrm{Hg}$.

$\mathrm{O} \mathrm{Hg}$ vem sendo utilizado como eletrodo na polarografia clássica desde a década de 20. ${ }^{7}$ Em 1922, Jaroslav Heyrovsky desenvolveu um método para determinar qualitativamente e quantitativamente várias substâncias em soluções usando medições elétricas. Heyrovsky utilizou o eletrodo gotejante de mercúrio (DME, do inglês dropping mercury electrode) como eletrodo de trabalho e chamou a técnica de polarografia. Essa descoberta, rendeu ao pesquisador em 1959, o Prêmio Nobel de Química por ter desenvolvido os métodos polarográficos de análise. ${ }^{8}$ A maior contribuição de Heyrovsky foi correlacionar, através de parâmetros experimentais otimizados, a corrente de um analito, em um potencial característico, à concentração do mesmo em uma solução aquosa, e possibilitar estudos eletroquímicos em sistemas que são irreversíveis. ${ }^{9}$

As primeiras medidas com eletrodo gotejante de mercúrio demandavam quantidades significativas de mercúrio metálico no decorrer de uma análise, devido ao constante fluxo de Hg. Nessas medidas eram alcançados elevados limites de quantificação em relação aos limites atuais. Contudo, ao longo dos anos, a necessidade de limites de detecção e de quantificação cada vez menores foi aumentando, com isso, a indispensabilidade de métodos instrumentais cada vez mais sensíveis. Na década de 70, ocorreu um crescimento no uso de técnicas de separação para melhorar a sensibilidade dos métodos analíticos, destacando-se a cromatografia gasosa (CG), seguida mais tarde pela cromatografia líquida (HPLC). Essas técnicas aliadas à sistemas de detecção são ideais para determinações de compostos orgânicos. Para os compostos inorgânicos, a espectrometria de absorção atômica (AAS) se tornou uma ferramenta valiosa muito utilizada. ${ }^{10}$ No entanto, a instrumentação necessária para determinações usando HPLC, CG e AAS é custosa. Além disso, processos de tratamento de amostra como pré-concentração e limpeza (cleanup) são usualmente empregados em especial para as técnicas cromatográficas. Tais desvantagens apresentadas, como os elevados custos, são menos latentes para as técnicas voltamétricas, uma vez que a instrumentação é relativamente de baixo custo e suprimentos não são requeridos, bem como a necessidade de pré-tratamentos da amostra, tantas vezes dispensáveis na voltametria. Atualmente, com o avanço 
tecnológico instrumental e a utilização do eletrodo de $\mathrm{Hg}$ no modo de gota pendente (HMDE), o volume de Hg gasto durante uma análise é consideravelmente menor em relação ao modo gotejante, diminuído o volume de resíduos gerados. É muito comum, observar apontamentos a respeito da toxicidade do $\mathrm{Hg}$ sem ao menos avaliar cientificamente as vantagens que o mesmo oferece nas determinações de um número imenso de analitos e matrizes complexas diferentes. Embora o $\mathrm{Hg}$, seja um elemento tóxico, as determinações empregando o HMDE dispensam, na maioria dos casos, a utilização de outros reagentes e solventes tóxicos. Além disso, tomados os devidos cuidados com o descarte dos resíduos gerados, riscos ambientais e ao analista são evitados. Este balanço entre benefícios alcançados e considerações negativas deve ser feito não só para o eletrodo de $\mathrm{Hg}$, mas também para outras técnicas analíticas.

No entanto, embora a voltametria com o HMDE fosse uma técnica consolidada, eletrodos alternativos de amálgama surgiram na tentativa de diminuir o volume de metal utilizado e melhorar a estabilidade mecânica do eletrodo de trabalho sem perder suas propriedades eletroquímicas. ${ }^{11,12}$

Eletrodos de amálgama como os de metais amalgamados, pasta de amálgama com e sem ligante líquido e eletrodos sólidos de amálgama são algumas variações do eletrodo de mercúrio. ${ }^{13}$ Cada um desses eletrodos apresenta vantagens e desvantagens. Os eletrodos sólidos de mercúrio se destacam por possuírem propriedades eletroquímicas semelhantes ao HMDE, porém, com uma superfície eletroquímica completamente sólida. ${ }^{13}$ Além disso, aplicações em sistemas em fluxo e in situ também são vantagens destes eletrodos. Uma variação do eletrodo sólido de amálgama consiste na modificação da superfície da amálgama com um filme de mercúrio. Este último eletrodo apresenta como vantagens uma maior estabilidade e melhor reprodutibilidade da superfície do filme quando comparado aos eletrodos de metais amalgamado, além de resposta similar ao tradicional eletrodo de $\mathrm{Hg} .{ }^{13}$

Embora os eletrodos de amálgama apresentem diversas vantagens e aplicações, a presença do $\mathrm{Hg}$ em sua composição impulsionou a busca por outros eletrodos com similares propriedades, tais como os de filme de bismuto, carbono vítreo, diamante dopado com boro, etc. ${ }^{14-17}$ Os eletrodos de filme de bismuto, por exemplo, possuem vantagens tais como baixa corrente residual, larga faixa de potencial de trabalho negativo, boa estabilidade química e mecânica, baixa interferência causada pelo oxigênio, além de serem mais ambientalmente amigáveis devido à baixa toxicidade do bismuto. Devido às qualidades apresentadas pelos eletrodos de filme de bismuto, estes têm sido frequentemente empregados em determinações de compostos orgânicos e inorgânicos..$^{18-22}$ Nesse sentido, Carvalho e colaboradores realizaram um estudo comparativo entre os eletrodos de filme de bismuto e filme de $\mathrm{Hg}$ como eletrodos de trabalho para a determinação simultânea de $\mathrm{Cd}, \mathrm{Pb}, \mathrm{Tl}$ e $\mathrm{Cu}$ em amostras salinas. Estes eletrodos foram ainda comparados com o HMDE na determinação dos metais. Dentre os eletrodos de trabalho, o eletrodo de filme de $\mathrm{Hg}$ se mostrou mais adequado para a determinação simultânea dos metais. Baixos limites de detecção (LD) foram alcançados e as determinações foram conduzidas em amostras altamente salinas não diluídas sem quaisquer interferências, sendo esta uma grande vantagem quando comparado às técnicas espectrométricas. ${ }^{12}$

Os eletrodos sólidos foram empregados com sucesso na determinação de diversos compostos em substituição ao HMDE. Além da resistência mecânica, a possibilidade de varredura em potencias positivos e a ausência do $\mathrm{Hg}$ líquido são vantagens destes eletrodos. Entretanto, a passivação da superfície e heterogeneidade da mesma frequentemente estão associadas, comprometendo a reprodutibilidade das análises voltamétricas. ${ }^{13}$ Sendo assim, o HDME continua sendo uma opção a ser considerada devido as suas propriedades eletroquímicas. Desta forma, uma ponderação entre os resultados que podem ser obtidos e os custos, reagentes e resíduos gerados deve ser realizada para a escolha da técnica ou instrumentação para as análises.

A preocupação em torno da toxicidade do $\mathrm{Hg}$ utilizado em análises voltamétricas deve estar sempre presente. Entretanto, usualmente, volumes inferiores a $1 \mu \mathrm{L}$ de $\mathrm{Hg}$ podem ser utilizados em uma determinação completa empregando HMDE. ${ }^{23}$ Essa quantidade necessária de mercúrio pode ser considerada desprezível frente a outras fontes de $\mathrm{Hg}$, tais como na produção de ouro, responsável pela liberação anual de toneladas de $\mathrm{Hg}$ ao ambiente em forma de vapores e no seu estado elementar. ${ }^{24-26}$ Zumam cita em seu artigo que "...vivemos em uma era de mercurofobia irracional”, pois o $\mathrm{Hg}$ metálico em temperatura ambiente é inócuo, nas pequenas quantidades usadas com o eletrodo no modo pendente. Os compostos organomercuriais apresentam uma toxicidade mais elevada, porém estes não são formados durante as análises eletroanalíticas. ${ }^{9}$ Embora Zumam afirme a ausência de riscos na utilização do $\mathrm{Hg}$ como eletrodo, cuidados para evitar a volatilização e descarte inadequado deste elemento são apreciados. Problemas à saúde do analista e contaminações ambientais são efeitos colaterais indesejados na pesquisa científica e aplicação empresarial, de um modo geral. No caso das técnicas voltamétricas que empregam eletrodos de trabalho com mercúrio, pode-se minimizar e evitar esses efeitos de saúde e contaminação ambiental, permitindo o total aproveitamento das vantagens que estes eletrodos podem oferecer.

Atualmente, os procedimentos de utilização do $\mathrm{Hg}$ junto com as técnicas pulsadas, como pulso diferencial e onda quadrada aliadas às técnicas de redissolução, permitiram à voltametria alcançar limites de quantificação comparáveis aos obtidos pelas técnicas cromatográficas e espectrométricas. Desta forma, a voltametria de redissolução empregando o HMDE é uma interessante ferramenta para a determinação de níveis traço de compostos orgânicos e íons metálicos em diversas amostras. A aplicação desta técnica de análise vai desde o controle de qualidade em produtos farmacêuticos ao monitoramento de espécies orgânicas e inorgânicas em compartimentos ambientais.

Outro fato relevante do emprego do HMDE refere-se à distribuição geográfica das publicações. Um levantamento bibliográfico realizado em diversos periódicos através de pesquisas no Science Direct, SciELO, PubMed, ferramenta de busca do Mendeley (Literature Search), University of Manitoba Libraries, Google Acadêmico e Google, com as palavras chave: eletrodo de mercúrio, HMDE, hanging mercury drop electrode, voltametria, voltammetry, determinação voltamétrica e voltammetric determination, resultou em 151 publicações nos últimos 10 anos. Os trabalhos encontrados foram então organizados levando em conta seu país de origem e notou-se que a distribuição global dos grupos de pesquisa que publicaram com este eletrodo abrange quase todos os continentes, e até mesmo países europeus continuam utilizando tal técnica de análise (Figura 1). Esta avaliação demonstra que mesmo existindo outras técnicas de análise mais sofisticadas que surgiram com o avanço tecnológico expressivo no campo científico, o HMDE não foi completamente abandonado. Entretanto, é possível notar que esta técnica de análise está sendo utilizada principalmente por países de terceiro mundo, onde os recursos financeiros para pesquisa são mais escassos e o uso de metodologias de baixo custo é priorizada.

Embora nesta revisão tenham sido encontradas publicações oriundas de vinte países, alguns destes representam parcelas expressivas na contribuição deste levantamento bibliográfico. Brasil, Índia e Turquia ocupam os primeiros lugares em número de publicações com HMDE, seguidos pela Arábia Saudita e Egito com 8,6 e 7,3\% de participação, respectivamente. A Figura 2 apresenta a colaboração de cada país para com estudos realizados com HMDE nos últimos dez anos avaliados no presente trabalho.

Os trabalhos científicos empregando eletrodo de gota pendente de $\mathrm{Hg}$ apresentados neste artigo pertencem a uma lacuna temporal 


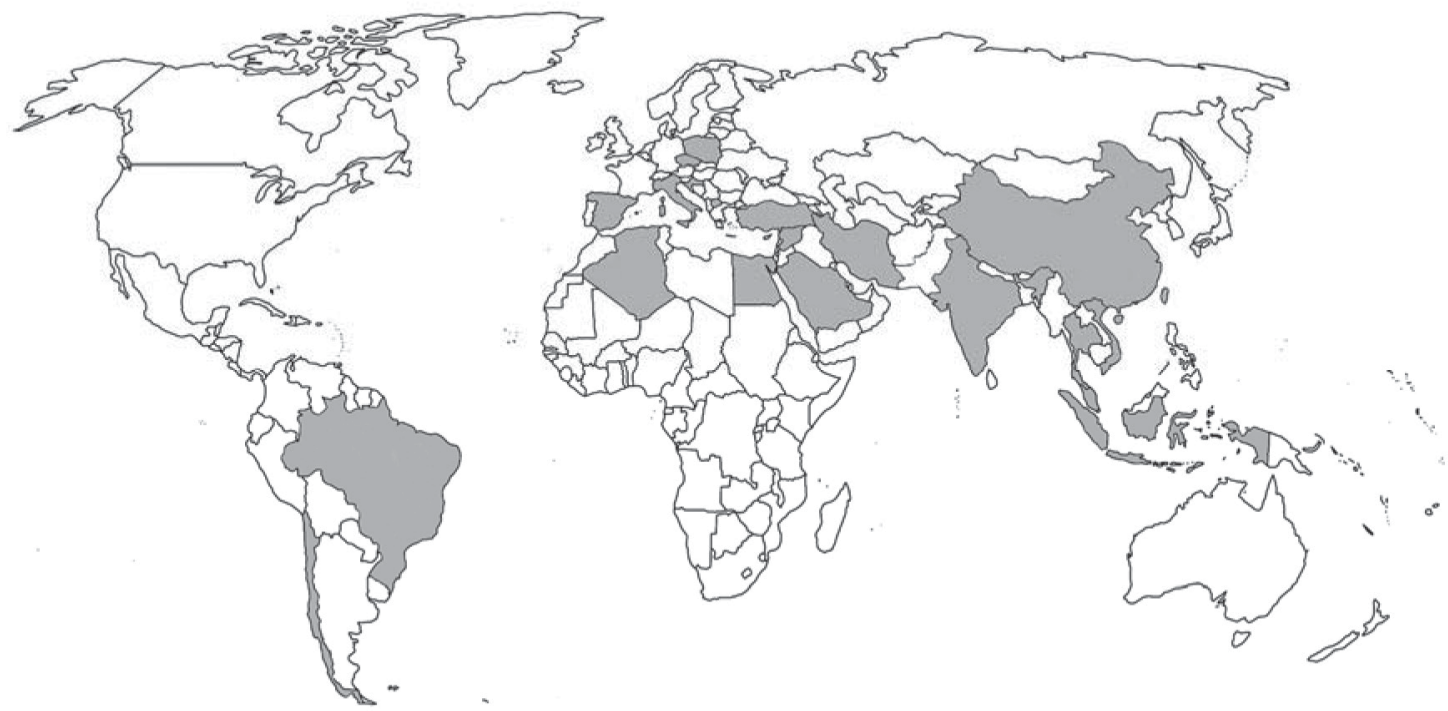

Figura 1. Distribuição geográfica de publicações empregando HMDE nos últimos dez anos. Em negrito países que publicaram empregando HMDE através da busca bibliográfica realizada em ferramentas de busca tais como o Science Direct, SciELO, PubMed, ferramenta de busca do Mendeley, University of Manitoba Libraries, Google Acadêmico e Google. Modificado de <http://www.estadosecapitaisdobrasil.com>, acessado 25.05.2017

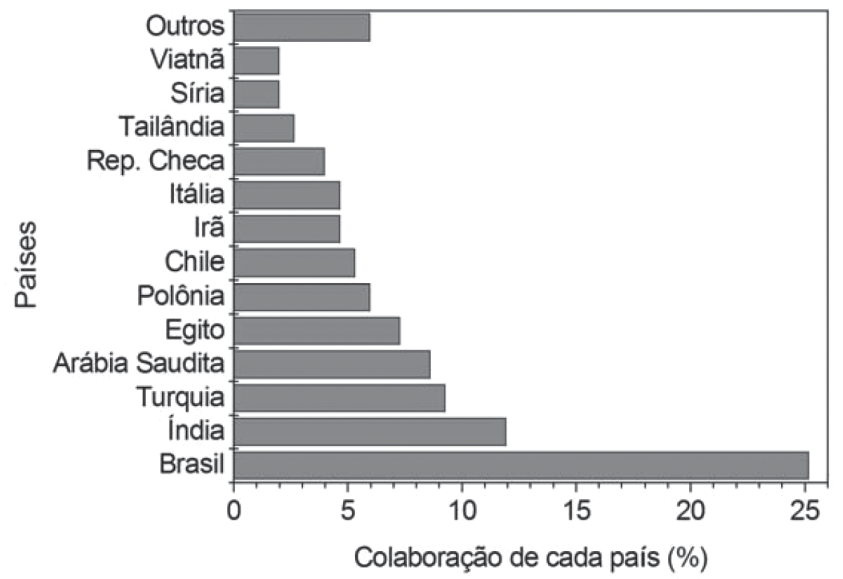

* China, Croácia, Argélia, Espanha, Indonésia, Macedônia e Malásia estão compiladas em "outros".

Figura 2. Contribuição de cada país na produção científica de trabalhos empregando HMDE através da busca bibliográfica realizada em ferramentas de busca tais como o Science Direct, SciELO, PubMed, ferramenta de busca do Mendeley, University of Manitoba Libraries, Google Acadêmico e Google

de dez anos. Artigos científicos publicados desde o ano de 2007 até 2016, foram apresentados nesta revisão com o intuito de elucidar as recentes aplicações, em especial do HMDE, em estudos de objetivos variados. Relacionando o número com a data de publicação, pode-se observar ausência de uma tendência que aponte para um decréscimo ou aumento da utilização desta técnica analítica nos últimos anos (Figura 3A). Nesta figura, nota-se que os anos compreendidos entre 2010 e 2014 apresentaram publicações acima da média, seguido por um decréscimo nos dois anos subsequentes. Entretanto, para o ano de 2016 foram encontrados mais artigos publicados que em 2009 por exemplo. A Figura 3B relaciona o número de publicações encontradas para cada ano avaliado, distinguindo entre estudos com compostos orgânicos e íons metálicos. Entre analitos orgânicos e inorgânicos, comportamentos similares foram observados em função do número de publicações por ano, tendo apenas em 2008, 2010 e 2013 mais trabalhos encontrados com íons metálicos. Porém, nestes últimos anos, de forma geral, foram encontrados mais trabalhos com fármacos, corantes, pesticidas, etc., do que com íons metálicos, que representaram apenas $40 \%$ do total.

Nesse contexto, fica claro que a voltametria empregando o HMDE continua sendo utilizada mesmo após o surgimento de técnicas analíticas mais avançadas e eletrodos alternativos. É natural que com o surgimento de outras técnicas, o número de publicações com HMDE reduza. Entretanto, os dados mostrados neste artigo apontam para o não abandono deste eletrodo. Sendo assim, o presente trabalho teve por objetivo mostrar que o HMDE continua tendo importância significativa na pesquisa científica, principalmente em países em desenvolvimento, onde recursos financeiros destinados à pesquisa não são tão abundantes. De forma geral, quando cuidados adequados são tomados durante a utilização e descarte do Hg, o HMDE apresenta qualidades indiscutíveis, podendo ser empregado em estudos variados.

\section{Determinação de íons metálicos usando o HMDE}

O eletrodo de $\mathrm{Hg}$ de gota pendente, $\mathrm{HMDE}$, apresenta grande aplicabilidade na determinação de espécies inorgânicas. Sua aplicação na determinação de íons metálicos é respeitada como uma técnica altamente sensível, exata e precisa, possibilitando quantificações de metais em níveis traço nas mais distintas amostras. A possibilidade de pré-concentração em uma forma de amálgama seguida pela redissolução da espécie de interesse, proporciona limites de quantificação consideravelmente baixos. Outra vantagem se relaciona com a possibilidade de obter informações sobre o estado de oxidação do analito e, portanto, sobre a especiação de um mesmo elemento. Nesse sentido, dependendo do método voltamétrico usado, é possível estimar a concentração de espécies metálicas livres ou espécies que formam complexos cineticamente reversíveis (espécies lábeis). Essas formas são consideradas lábeis e eletroativas no HMDE, enquanto que as espécies que formam complexos são consideradas inertes..$^{27,28}$

Portanto, nas medidas voltamétricas com HMDE, as espécies que podem ser determinadas devem ser eletroativas, isto é, sofrem processos de redução ou oxidação dento da faixa de potencial adequado para esse eletrodo de trabalho ou, ainda, devem ser adsorvidas no eletrodo para métodos voltamétricos específicos. Também é possível determinar analitos inertes após a sua conversão em 

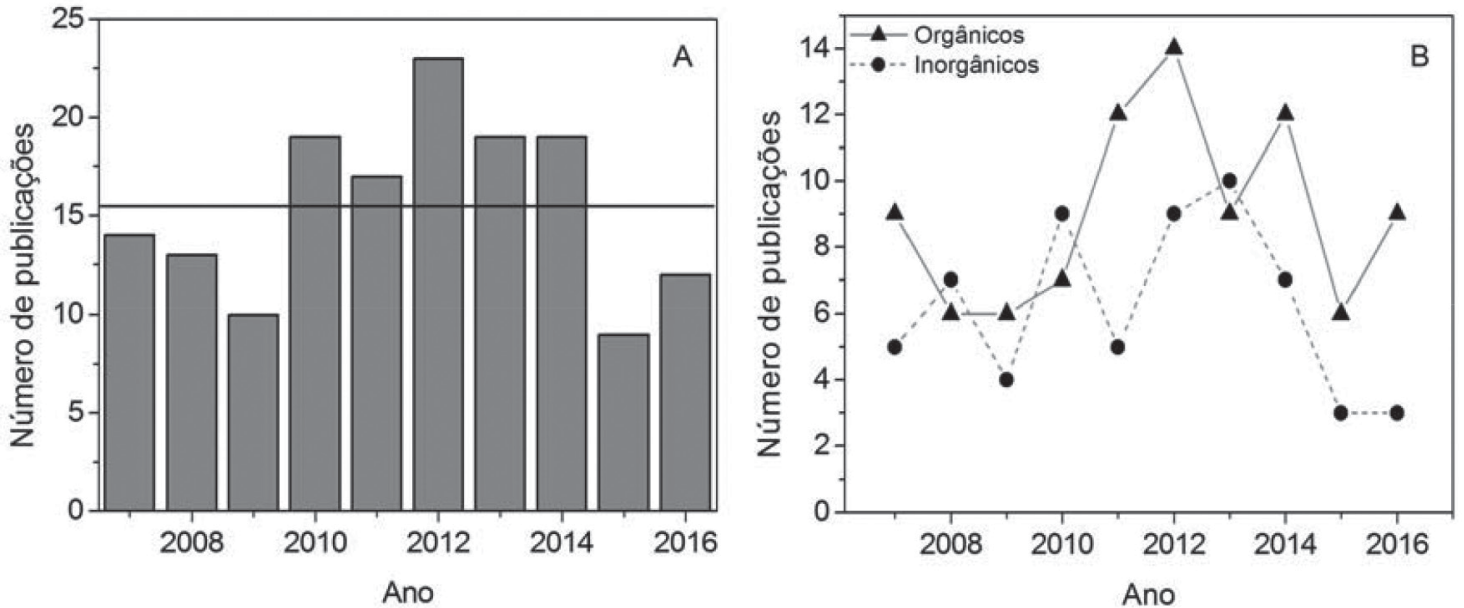

Figura 3. Gráficos relativos ao número de publicações por ano empregando HMDE. (A) Publicações totais. (B) Segregação entre publicações com compostos orgânicos e inorgânicos

espécies eletroativas, por exemplo, através da sua complexação com ligantes. A maioria dos protocolos analíticos desenvolvidos com a voltametria e HMDE são empregados em meio aquoso, porém, é possível ainda serem realizados em meios não aquosos. ${ }^{29,30}$ Contudo, as espécies eletroativas devem ser solúveis em um solvente que deve ser condutor. ${ }^{9}$ Vantagens como maior faixa de potencial e oxidação do $\mathrm{Hg}$ em potencias mais positivos, além da maior solubilidade de algumas amostras e analitos, podem ser citados para determinações em meio não aquoso. ${ }^{30} \mathrm{O}$ HMDE permite, dentre outras aplicações, a avaliação da presença de íons metálicos em amostras ambientais, farmacêutica, toxicológica, nutricional, etc. A determinação de íons metálicos em solos, águas e em organismos vivos expostos a contaminação é frequente. ${ }^{31-34}$ Repula et al. estudaram a presença de $\mathrm{Cr}$ e $\mathrm{Pb}$ em briófitas e pteridófitas coletadas em um córrego que recebe descarte de curtume. O HMDE e técnica de pulso diferencial foram utilizados para as determinações dos íons metálicos. Concentrações elevadas destes metais que foram determinadas nas amostras podem indicar uma contaminação ambiental no ponto de coleta. ${ }^{31}$ Repula et al., em outro trabalho, determinaram as concentrações de $\mathrm{Cr}$ e $\mathrm{Pb}$ em tecidos de peixes (musculo e fígado) do mesmo córrego empregando o HMDE como eletrodo de trabalho. No trabalho é relatado que os metais bioacumularam preferencialmente no fígado dos peixes e as concentrações encontradas foram maiores que as permitidas para o consumo humano. ${ }^{32}$

Estudos com $\mathrm{As}, \mathrm{Cu}, \mathrm{Pb}, \mathrm{Cd}$ e $\mathrm{Zn}$ foram realizados na tentativa de determinar a concentração destes elementos em plantas possivelmente contaminadas. ${ }^{35,36}$ Locatelli e Melucci desenvolveram um método sequencial de duas etapas para a determinação de $\mathrm{Hg}, \mathrm{Cu}$, $\mathrm{Pb}, \mathrm{Cd}$ e $\mathrm{Zn}$ em amostras de vegetais como espinafre, alface e tomate empregando um eletrodo de ouro e o HMDE. O HMDE foi utilizado na determinação do $\mathrm{Cu}, \mathrm{Pb}, \mathrm{Cd}$ e $\mathrm{Zn}$ e o eletrodo de ouro empregado na determinação de $\mathrm{Hg}$ e $\mathrm{Cu}$. $\mathrm{O}$ método mostrou-se adequado e tratamentos da amostra foram desnecessários. Uma comparação entre os resultados obtidos entre os vegetais lavados e não lavados mostrou uma maior concentração de espécies metálicas nas amostras não higienizadas. A concentração determinada de $\mathrm{Pb}$ e $\mathrm{Cd}$ foi maior que a permitida por lei..$^{35}$

A presença de metais em ambientes aquáticos pode ter origem natural ou então pode ser um indício de possível contaminação antrópica devido descartes inadequados e utilização de defensivos agrícolas. A preocupação com este compartimento ambiental é significativa, devido aos possíveis riscos aos quais organismos aquáticos estão expostos..$^{33,34,37-43}$ No mesmo sentido, alguns trabalhos também avaliaram a presença de metais em águas de torneira e água envasada. ${ }^{44-49}$
Arab and Alshikh avaliaram a presença de $\mathrm{Cd}, \mathrm{Cu}, \mathrm{Fe}, \mathrm{Pb} \mathrm{Zn}, \mathrm{Se}$ em amostras de águas de torneira de diferentes pontos da cidade de Jeddah na Arábia Saudita, durante um ano empregando voltametria de redissolução por pulso diferencial e HMDE como eletrodo de trabalho. Várias amostras coletadas apresentaram concentrações elevadas dos metais estudados, porém, consideradas aceitáveis pela Organização Mundial da Saúde. ${ }^{44}$ Raj e colaboradores analisaram amostras de água de torneira de Delhi (Índia) empregando voltametria de redissolução anódica por pulso diferencial. ${ }^{47}$ Rojas e colaboradores desenvolveram um método eletroquímico para a determinação de concentrações traço de $\mathrm{Sb}(\mathrm{III})$ na presença do ácido quercetina-5-sulfônico em amostras de água. Estudos de recuperação em água ultrapura, de água mineral e água de torneira provaram a aplicabilidade do método. Amostras de água de torneira apresentaram concentração de $3.59 \pm 0.09 \mu \mathrm{g} \mathrm{L}^{-1}$ de $\mathrm{Sb}(\mathrm{III}) .^{48}$

Embora a aplicação dos métodos voltamétricos com HMDE ocorra de forma bem ampla para amostras de água natural, é possível encontrar trabalhos publicados para outras amostras de interesse ambiental, como o ar e solo. ${ }^{34,37,41,50}$ Catrambone e colaboradores otimizaram e validaram um método baseado na extração de Cr solúvel, com auxílio de ultrassom de amostras de material particulado atmosférico para determinação de $\mathrm{Cr}$ (III) e $\mathrm{Cr}$ (VI). Voltametria adsortiva de redissolução e ácido dietileno triamino pentaacético como complexante na presença de nitrato foram utilizados nas determinações. A especiação do Cr foi possível e a análise das amostras coletadas em sítios industrias indicou significativa presença de $\mathrm{Cr}(\mathrm{VI})$. Para as áreas rurais, os teores de $\mathrm{Cr}(\mathrm{VI})$ foram abaixo do limite de detecção. Os autores relatam que o $\mathrm{Cr}(\mathrm{VI})$ permanece principalmente no material particulado fino, e possui origem majoritariamente industrial, enquanto que o $\mathrm{Cr}$ (III) apresentou distribuição bimodal, já que possui origem antropogênica e natural. ${ }^{50}$

Devido à versatilidade da voltametria e baixa ou inexistente necessidade de tratamento de amostra, diferentes matrizes além das ambientais pertencem ao escopo que podem ser estudadas pela técnica. Amostras como composições farmacêuticas, ${ }^{51-54}$ fluidos biológicos, ${ }^{55,56}$ alimentos, ${ }^{57-62}$ entre outras, foram estudadas. ${ }^{40,63,64} \mathrm{~A}$ grande versatilidade da voltametria para com a determinação de íons metálicos em distintas matrizes encontra-se apresentada na Tabela 1.

Como pode ser visto na Tabela 1 , diferentes amostras podem ser analisadas empregando voltametria com o HMDE como eletrodo de trabalho na determinação de íons metálicos. Alguns trabalhos empregaram um agente complexante ou ligante para possibilitar ou otimizar a determinação por voltametria. Limites de detecção na ordem de partes por bilhão podem ser considerados comparáveis aos obtidos por técnicas espectrométricas. A faixa linear de trabalho 
Tabela 1. Uso da voltameria e HMDE como eletrodo de trabalho na determinação de íons metálicos

\begin{tabular}{|c|c|c|c|c|c|c|}
\hline Analito & Ligante & Amostra & Método & LD & Faixa linear & Ref. \\
\hline $\mathrm{Zn}$ & alizarina & café instantâneo & $\mathrm{CV}, \mathrm{SW}-\mathrm{CSV}$ & $1 \times 10^{-8} \mathrm{~mol} \mathrm{~L}^{-1}$ & $\begin{array}{c}5 \times 10^{-8}-4 \times 10^{-7} \\
\mathrm{~mol} \mathrm{~L}^{-1}\end{array}$ & 57 \\
\hline $\mathrm{Cd}, \mathrm{Cu}, \mathrm{Fe}, \mathrm{Pb}, \mathrm{Zn}, \mathrm{Se}$ & trietanolamina & água de torneira & DP - ASV, CSV & NI & NI & 44 \\
\hline $\mathrm{Zn}$ & 8- hidroxiquinolina & água marinha & SW - SV & $0,05 \mu \mathrm{g} \mathrm{L}^{-1}$ & $0,1-48,0 \mu g \mathrm{~L}^{-1}$ & 33 \\
\hline $\mathrm{Cu}$ & $\begin{array}{l}\text { benzenossulfonil- } \\
\text { hidrazida }\end{array}$ & sangue & DP - CSV & $0,186 \mathrm{ng} \mathrm{mL}^{-1}$ & $0,62-275 \mathrm{ng} \mathrm{mL}^{-1}$ & 56 \\
\hline $\mathrm{Cd}, \mathrm{Cu}, \mathrm{Pb}, \mathrm{Zn}$ & & sedimentos, peixes, aves & DP - ASV & $0,014-0,021 \mathrm{mg} \mathrm{kg}^{-1}$ & $0,005-1.0 \mathrm{mg} \mathrm{kg}^{-1}$ & 41 \\
\hline $\mathrm{Pd}$ & SQ-OH & poeira de estrada, água natural & $\mathrm{CV}, \mathrm{CSV}$ & $0,05 \mathrm{ng} \mathrm{mL}^{-1}$ & $0,2-32,5 \mathrm{ng} \mathrm{mL}^{-1}$ & 37 \\
\hline $\mathrm{Cu}, \mathrm{As}$ & & poste de energia & DP - ASV, DP - CSV & $\mathrm{mg} \mathrm{kg}^{-1}$ & NI & 63 \\
\hline $\mathrm{Zn}, \mathrm{Ni}$ & azofloxina & estudo eletroquímico & $\mathrm{CV}, \mathrm{SW}$ & NI & NI & 65 \\
\hline $\mathrm{Al}$ & DASA & erva mate & $\mathrm{CV}, \mathrm{DP}-\mathrm{CSV}$ & $0,5 \mu \mathrm{g} \mathrm{L}^{-1}$ & $1,7-100 \mu \mathrm{g} \mathrm{L}^{-1}$ & 62 \\
\hline $\mathrm{Cr}(\mathrm{III}), \mathrm{Cr}(\mathrm{VI})$ & $\begin{array}{l}\text { ácido dietileno tri- } \\
\text { amino pentaacético }\end{array}$ & matéria particulada atmosférica & SV & 1,$8 ; 2,6 \mathrm{ng} /$ filtro & NI & 50 \\
\hline As(III), TAs & $\mathrm{Cu}(\mathrm{II})$ & plantas & SW - CSV & 0,$5 ; 0,4 \mu \mathrm{g} \mathrm{L}^{-1}$ & $10-1000 \mu \mathrm{g} \mathrm{L}{ }^{-1}$ & 36 \\
\hline Sn & & kit de radiofármacos & DP & $3 \mathrm{mg} \mathrm{L}^{-1}$ & $10-50 \mathrm{mg} \mathrm{L}^{-1}$ & 66 \\
\hline $\mathrm{Zn}, \mathrm{Cd}, \mathrm{Pb} \mathrm{Cu}$ & & efluente petroquímico & DP - ASV & $1,5-4,4 \mu \mathrm{g} \mathrm{L}^{-1}$ & NI & 67 \\
\hline $\mathrm{Cd}, \mathrm{Cu}, \mathrm{Pb}, \mathrm{Zn}$ & & $\begin{array}{l}\text { água natural, urina, fluidos pós- } \\
\text { hemodiálise }\end{array}$ & ASV & $0,1-2,0 \mu \mathrm{g} \mathrm{L}-1$ & NI & 55 \\
\hline $\mathrm{Cd}, \mathrm{Tl}, \mathrm{Pb}, \mathrm{Cu}$ & & $\begin{array}{l}\text { água marinha, fluidos hidrotérmi- } \\
\text { cos, concentrados de diálise }\end{array}$ & ASV & NI & NI & 12 \\
\hline $\mathrm{Al}, \mathrm{Cr}, \mathrm{Ni}$ & $\begin{array}{l}\text { violeta de solocromo } \\
\text { RS, ácido dietileno } \\
\text { triamino pentaacético, } \\
\text { dimetilglioxima }\end{array}$ & formulações farmacêuticas & DP - CSV & $1,2-19 \mathrm{ng} \mathrm{mL}^{-1}$ & $0-200 \mu \mathrm{g} \mathrm{L}-1$ & 68 \\
\hline As & $\mathrm{Cu}, \mathrm{Se}$ & águas naturais & SW - CSV & NI & NI & 43 \\
\hline $\mathrm{Cu}, \mathrm{Cd}, \mathrm{Pb}, \mathrm{Zn}$ & & águas naturais & DP - ASV & $0,05 \mu \mathrm{g} \mathrm{L}^{-1}$ & NI & 27 \\
\hline $\mathrm{As}(\mathrm{III}), \mathrm{As}(\mathrm{V})$ & SDDC & água, soluções salinas & DP - CSV & $0,3-15 \mu \mathrm{g} \mathrm{L}^{-1}$ & $10-100 \mu \mathrm{g} \mathrm{L}^{-1}$ & 69 \\
\hline $\mathrm{As}(\mathrm{III}), \mathrm{As}(\mathrm{V})$ & & sucos & $\mathrm{DP}-\mathrm{CSV}$ & $0,08 \mu \mathrm{g} \mathrm{L}^{-1}$ & $1-5 \mu \mathrm{g} \mathrm{L}^{-1}$ & 70 \\
\hline $\mathrm{H}_{2} \mathrm{~S}, \mathrm{HS}^{-}, \mathrm{S}^{-2}$ & espécies de enxofre & fluidos hidrotermais & DP - CSV & $0,3 \mu \mathrm{mol} \mathrm{L}^{-1}$ & $0,5-4,0 \mu \mathrm{mol} \mathrm{L}^{-1}$ & 71 \\
\hline $\mathrm{Sb}(\mathrm{III}), \mathrm{Sb}(\mathrm{V})$ & $\begin{array}{l}\text { 4-(2- tiazolilazo })- \\
\text { resorcinol } \\
\end{array}$ & águas naturais & $\mathrm{CV}, \mathrm{DP}-\mathrm{CSV}$ & $0,05 \mu \mathrm{g} \mathrm{L}-1$ & $0,17-12 \mu \mathrm{g} \mathrm{L}-1$ & 38 \\
\hline $\mathrm{Sb}, \mathrm{Pb}$ & catecol & resíduos de arma de fogo & DP, SW - CSV & $0,58-1,5 \mathrm{n} \mathrm{mol} \mathrm{L}-1$ & $2,0-710,0 \mathrm{n} \mathrm{mol} \mathrm{L^{-1 }}$ & 72 \\
\hline As & & águas naturais & DP & $2,7 \mu \mathrm{g} \mathrm{L}^{-1}$ & $0,049-14,68 \mathrm{mg} \mathrm{L}^{-1}$ & 39 \\
\hline $\mathrm{Cu}$ & calceína azul & $\begin{array}{l}\text { reagentes de grau analítico, água } \\
\text { de torneira e mineral, medica- } \\
\text { mentos }\end{array}$ & $\mathrm{CV}, \mathrm{DP}-\mathrm{CSV}$ & $0,01 \mathrm{ng} \mathrm{mL}^{-1}$ & $0,02-15 \mathrm{ng} \mathrm{mL}^{-1}$ & 73 \\
\hline $\mathrm{Cu}, \mathrm{Cd}$ & ácido ditiosalicílico & águas naturais, chá & DP - CSV & 0,$5 ; 0,3 \mathrm{ng} \mathrm{mL}^{-1}$ & $\begin{array}{c}1-80 ; 1-240 \mathrm{ng} \\
\mathrm{mL}^{-1}\end{array}$ & 40 \\
\hline $\mathrm{Sb}(\mathrm{III}), \mathrm{Sb}(\mathrm{V})$ & pirogalol & $\begin{array}{l}\text { formulações farmacêuticas, água } \\
\text { de torneira }\end{array}$ & CV, DP - SV & $\begin{array}{c}1,03 \times 10^{-10} ; 9,48 \times \\
10^{-9} \mathrm{~mol} \mathrm{~L}^{-1}\end{array}$ & $4,97-26,76 \mathrm{n} \mathrm{mol} \mathrm{L}-1$ & 45 \\
\hline $\mathrm{Se}, \mathrm{Cd}, \mathrm{Pb}$ & & formulações farmacêuticas & DP - ASV & $0,08-0,66 \mu \mathrm{g} \mathrm{mL}-1$ & $0,5-8,5 \mathrm{ng} \mathrm{mL}^{-1}$ & 52 \\
\hline $\mathrm{As}(\mathrm{III}), \mathrm{As}(\mathrm{V})$ & $\mathrm{Cu}(\mathrm{II})$ & água, solo, minérios & CSV & $0,3 \mathrm{mgL}^{-1}$ & $0,3-50 \mathrm{mg} \mathrm{L}^{-1}$ & 34 \\
\hline $\begin{array}{l}\mathrm{Cu}, \mathrm{Cr}, \mathrm{Tl}, \mathrm{Pb}, \mathrm{Sn}, \mathrm{Sb}, \\
\mathrm{Zn}\end{array}$ & & farinhas, cereais, solos & SW - ASV & $0,011-0,103 \mu \mathrm{g} \mathrm{g}^{-1}$ & NI & 74 \\
\hline $\mathrm{Cu}, \mathrm{Pb}, \mathrm{Cd}, \mathrm{Zn}$ & & farinhas & SW - ASV & $0,0031-0,1 \mu \mathrm{g} \mathrm{L}{ }^{-1}$ & NI & 59 \\
\hline $\mathrm{Cu}, \mathrm{Pb}, \mathrm{Cd}, \mathrm{Zn}$ & & vegetais & SW - ASV & $0,0043-0,23 \mu \mathrm{g} \mathrm{L}{ }^{-1}$ & NI & 35 \\
\hline $\mathrm{Zn}$ & & leite & & $0,02 \mathrm{ppm}$ & $0,4-2,6 \mathrm{ppm}$ & 75 \\
\hline $\begin{array}{l}\mathrm{Zn}, \mathrm{Cr}, \mathrm{Cu}, \mathrm{Sb}, \mathrm{Sn}, \mathrm{Pb} \text {, } \\
\mathrm{Fe}, \mathrm{Mn}, \mathrm{Mo}\end{array}$ & & farinhas & SW - ASV, SWV & $0,002-0,27 \mu \mathrm{g} \mathrm{L}^{-1}$ & $\mathrm{LD}-1,0 \mathrm{mg} \mathrm{L}^{-1}$ & 58 \\
\hline $\mathrm{Cu}, \mathrm{Pb}, \mathrm{Cd}, \mathrm{Zn}$ & & plantas medicinais & SW - ASV & $0,0041-0,25 \mu \mathrm{g} \mathrm{L} \mathrm{L}^{-1}$ & NI & 76 \\
\hline $\mathrm{Zn}$ & & leite & DP - ASV & NI & NI & 77 \\
\hline $\begin{array}{l}\mathrm{Al}, \mathrm{Fe}, \mathrm{Mo}, \mathrm{Zn}, \mathrm{Cd}, \mathrm{Pb}, \\
\mathrm{Cu}, \mathrm{NI}, \mathrm{Co}, \mathrm{Tl}, \mathrm{U}, \mathrm{V}, \mathrm{Cr}\end{array}$ & $\begin{array}{l}\text { SVRS, DTPA, CA, } \\
\text { DMG, oxine, }\end{array}$ & $\begin{array}{l}\text { fertilizantes, vegetais, produtos } \\
\text { alimentícios }\end{array}$ & DP & $0,61-3,82 \mu \mathrm{g} \mathrm{L}{ }^{-1}$ & $5-250 \mu \mathrm{g} \mathrm{L}^{-1}$ & 78 \\
\hline $\mathrm{Cd}, \mathrm{Pb}$ & $\begin{array}{c}\text { ácido quercetina- } \\
\text { 5-sulfônico } \\
\end{array}$ & água de torneira & SW - ASV & 0,$1 ; 0,3 \mu \mathrm{g} \mathrm{L} \mathrm{L}^{-1}$ & $0,5-45,0 \mu \mathrm{g} \mathrm{L}^{-1}$ & 46 \\
\hline In & $\mathrm{N}$ - metiltioureia & estudo eletroquímico & SW & $10^{-7} \mathrm{~mol} \mathrm{~L}^{-1}$ & $\begin{array}{c}1 \times 10^{-6}-5 \times 10^{-4} \\
\mathrm{~mol} \mathrm{~L}^{-1}\end{array}$ & 79 \\
\hline $\mathrm{Al}, \mathrm{Fe}$ & SVRS & fluido de pós-hemodiálise & DP - CSV & 1,$4 ; 1,8 \mu \mathrm{g} \mathrm{L}^{-1}$ & $5-200 \mu \mathrm{g} \mathrm{L}^{-1}$ & 80 \\
\hline $\mathrm{Se}(\mathrm{IV}), \mathrm{Se}(\mathrm{VI})$ & & $\begin{array}{l}\text { água marinha, fluidos hidrotérmi- } \\
\text { cos, concentrados de diálise }\end{array}$ & DP - CSV & $0,03 \mu \mathrm{g} \mathrm{L}^{-1}$ & $1-100 \mu \mathrm{g} \mathrm{L}^{-1}$ & 81 \\
\hline
\end{tabular}


Tabela 1. Uso da voltameria e HMDE como eletrodo de trabalho na determinação de íons metálicos (cont.)

\begin{tabular}{|c|c|c|c|c|c|c|}
\hline Analito & Ligante & Amostra & Método & LD & Faixa linear & Ref. \\
\hline $\mathrm{Cr}, \mathrm{Tl}, \mathrm{Cd}, \mathrm{Pb}, \mathrm{Cu}, \mathrm{Sb}$ & DTPA & $\begin{array}{c}\text { concentrados polieletrolíticos para } \\
\text { hemodiálise }\end{array}$ & $\begin{array}{l}\text { CV, AC, DP - ASV, } \\
\text { CSV }\end{array}$ & $0,03-0,27 \mu \mathrm{g} \mathrm{L}^{-1}$ & $0,14-600 \mu \mathrm{g} \mathrm{L}-1$ & 53 \\
\hline $\mathrm{Cu}, \mathrm{Pb}, \mathrm{Cd}, \mathrm{Ni}, \mathrm{Co}, \mathrm{Zn}$ & $\begin{array}{c}\text { dimetilglioxima, } \\
\text { 8-hidroxiquinolina }\end{array}$ & bioetanol & DP - SV & $0,0053-0,63 \mu \mathrm{g} \mathrm{L}-1$ & $0,03-10,4 \mu \mathrm{g} \mathrm{L}-1$ & 82 \\
\hline$\underline{\mathrm{Zn}}$ & & biológica & DP - ASV & NI & NI & 83 \\
\hline $\mathrm{Mn}$ & $\mathrm{Zn}(\mathrm{II})$ & solo & DP - ASV & $\mathrm{NI}$ & $\mathrm{NI}$ & 84 \\
\hline $\mathrm{Cd}, \mathrm{Cu}, \mathrm{Pb}, \mathrm{Zn}$ & & água de torneira & DP - ASV & NI & NI & 47 \\
\hline $\mathrm{Cr}, \mathrm{Pb}$ & & peixes & DP - ASV, CSV & 0,$03 ; 0,01 \mu \mathrm{g} \mathrm{g}^{-1}$ & $\mathrm{NI}$ & 32 \\
\hline $\mathrm{Sb}$ & $\begin{array}{l}\text { ácido quercetina- } \\
\text { 5-sulfônico }\end{array}$ & água de torneira e envasada & SW - ASV & $1.6 \mathrm{ng} \mathrm{L}^{-1}$ & $\mathrm{LD}-1.5 \mu \mathrm{g} \mathrm{L}-1$ & 48 \\
\hline $\mathrm{Sb}, \mathrm{Mo}$ & quercetina Q & água natural & SW - SV & 0,$076 ; 0,086 \mu \mathrm{g} \mathrm{L}^{-1}$ & $1,0-10,0 \mu \mathrm{g} \mathrm{L}^{-1}$ & 42 \\
\hline $\mathrm{V}$ & $\begin{array}{l}\text { ácido quercetina- } \\
\text { 5-sulfônico }\end{array}$ & $\begin{array}{l}\text { águas naturais, de torneira, puri- } \\
\text { ficada e envasada }\end{array}$ & $\mathrm{DP}-\mathrm{SV}$ & $0.23 \mathrm{ng} \mathrm{L}^{-1}$ & $\mathrm{LD}-0,35 \mu \mathrm{g} \mathrm{L}-1$ & 85 \\
\hline$\underline{\mathrm{Sb}}$ & vermelho de alizarina & água de torneira e envasada & DP - ASV & $1,45 \mu \mathrm{g} \mathrm{L}{ }^{-1}$ & $4,8-30 \mathrm{mg} \mathrm{L}^{-1}$ & 49 \\
\hline $\mathrm{Pb}, \mathrm{Cd}, \mathrm{Cu}, \mathrm{Zn}, \mathrm{Se}$ & & leite, derivados de leite & DP - ASV, CSV & $0,04-0,25 \mathrm{ppb}$ & $0,1-500 \mathrm{ppb}$ & 86 \\
\hline $\mathrm{CuS}$ & & águas naturais & SW - CSV & NI & NI & 87 \\
\hline $\mathrm{As}, \mathrm{Pb}$ & & organismos marinhos & ASV & NI & $\mathrm{NI}$ & 88 \\
\hline $\mathrm{Cu}$ & & águas naturais & DP - ASV & NI & NI & 89 \\
\hline $\mathrm{Cd}, \mathrm{Cu}, \mathrm{Pb}, \mathrm{Zn}$ & & alimentos fermentados & DP - ASV & $0,5-2,0 \mu \mathrm{g} \mathrm{L}^{-1}$ & $0-50 \mu \mathrm{g} \mathrm{L}^{-1}$ & 60 \\
\hline $\mathrm{Cu}, \mathrm{Zn}$ & & águas naturais & DP - ASV & 1,$8 ; 2,1 \mathrm{n} \mathrm{mol} \mathrm{L}^{-1}$ & NI & 28 \\
\hline $\mathrm{Cd}, \mathrm{Cu}, \mathrm{Pb}, \mathrm{Zn}$ & & gasolina & ASV & $8,58 \times 10^{-4}-0,87 \mu \mathrm{g} \mathrm{L}^{-1}$ & $7,89-61,20 \mathrm{n} \mathrm{mol} \mathrm{L}-1$ & 64 \\
\hline $\mathrm{Pb}$ & 8-hidroxiquinolona & peixe enlatado & SW - CSV & $0,108 \mu \mathrm{g} \mathrm{L} \mathrm{L}^{-1}$ & $0,5-90,0 \mu \mathrm{g} \mathrm{L}^{-1}$ & 61 \\
\hline $\mathrm{Al}$ & calon & fluido de diálise & CSV & $0,32 \mathrm{ng} \mathrm{mL}^{-1}$ & LD - 8,0 ng mL $\mathrm{mL}^{-1}$ & 90 \\
\hline $\mathrm{Sb}(\mathrm{III}), \mathrm{Sb}(\mathrm{V})$ & pirogalol & formulações & Varredura linear & 1,$2 ; 2,8 \mathrm{ng} \mathrm{mL}^{-1}$ & $3,0-240,0 \mathrm{ng} \mathrm{mL}^{-1}$ & 91 \\
\hline
\end{tabular}

$\mathrm{AC}=$ corrente alternada; $\mathrm{ASV}=$ voltametria de redissolução anódica; $\mathrm{CSV}=$ voltametria de redissolução catódica; $\mathrm{CV}=$ voltametria cíclica; $\mathrm{DP}=$ pulso diferencial; $\mathrm{NI}=$ não informado; $\mathrm{SV}=$ voltametria de redissolução; $\mathrm{SW}=$ onda quadrada.

alcançada é variável com o método voltamétrico empregado. Os métodos nos quais são empregados tempos de pré-concentração do analito (métodos de redissolução) são frequentemente empregados especialmente para analitos presentes em baixas concentrações, possibilitando minimizar os limites de detecção.

\section{Determinação de compostos orgânicos usando HMDE}

Determinações quantitativas de compostos orgânicos em matrizes complexas empregando métodos voltamétricos que usam como eletrodo de trabalho o HMDE são amplamente realizadas para amostras ambientas, farmacêuticas, etc. As vantagens já são conhecidas como adequada frequência analítica, medidas de baixo custo, etc. Além disso, outro aspecto vantajoso é a possibilidade de realizar as medidas voltamétricas de modo direto, sem a necessidade de procedimentos prévios de separação e extração do analito. Contudo, é conhecido que a seletividade da voltametria para compostos orgânicos pode ser mais limitada, sendo em alguns casos necessário aliar os procedimentos de extração, separação e cleanup previamente às medidas voltamétricas. Esses aspectos são mais importantes para as amostras ambientais, especialmente pela presença de matéria orgânica dissolvida, que é um interferente reconhecido. Nesse contexto, a voltametria pode ser empregada como uma ferramenta de "varredura" (screening) para avaliar a presença de compostos alvo, produtos de degradação e/ou metabólitos de maneira rápida e barata. Atualmente existem alguns trabalhos publicados que empregam a voltametria com esse tipo de abordagem..$^{92-95}$

Volke fez um levantamento bibliográfico de aplicações e vantagens da polarografia e voltametria em relação a outras técnicas de análise comumente utilizadas para estudos na química farmacêutica e farmacológica. O grande número de sítios ativos (reduzíveis ou oxidáveis) de compostos orgânicos, a possibilidade de determinação simultânea de diferentes substâncias, fácil preparação de amostra, a ausência de interferências oriundas por coloração ou turbidez de amostras, baixos limites de quantificação e volumes reduzidos de amostra são vantagens elencadas no que se trata da determinação de compostos farmacêuticos.?

O HMDE vem sendo utilizado na determinação de compostos orgânicos em amostras ambientais, biológicas e comerciais. Sua aplicação na determinação de fármacos permeia desde o controle de qualidade, até estudos ambientais, farmacocinéticos e forenses. Embora não seja a técnica de referência para fármacos, a determinação destes compostos em composições farmacêuticas é frequente e apresenta grande importância na avaliação da estabilidade das drogas bem como na determinação da concentração do princípio ativo nos medicamentos. ${ }^{54,96,97}$ Várias classes de fármacos podem ser estudadas empregando eletrodos de Hg. Antibióticos, ${ }^{98}$ anti-inflamatórios, ${ }^{99}$ fármacos psicoativos, ${ }^{92}$ antiparasitários, ${ }^{97}$ antieméticos, ${ }^{100}$ entre outros. Grupos nitro, amino, duplas e triplas ligações entre carbonos, etc., são centros eletroativos responsáveis pela resposta voltamétrica dos fármacos frente a aplicação de potencial. ${ }^{101}$ Alguns mecanismos de reações envolvendo a redução eletroquímica de fármacos no HMDE estão listados na Tabela 2.

Tabela 2. Reações eletroquímicas de fármacos no HMDE

\begin{tabular}{lc}
\hline Mecanismo & Ref. \\
\hline $\mathrm{Hg}\left[\mathrm{R}-\mathrm{NO}_{2}\right]_{\text {ads }}+4 \mathrm{e}^{-}+4 \mathrm{H}^{+} \rightarrow \mathrm{Hg}+[\mathrm{R}-\mathrm{NH}-\mathrm{OH}]+\mathrm{H}_{2} \mathrm{O}$ & 54 \\
$\mathrm{Hg}[\mathrm{R}-\mathrm{C}=\mathrm{N}-\mathrm{R}]_{\text {ads }}+2 \mathrm{e}^{-}+2 \mathrm{H}^{+} \rightarrow \mathrm{Hg}+[\mathrm{R}-\mathrm{CH}-\mathrm{NH}-\mathrm{R}]$ & 82 \\
$\mathrm{Hg}[\mathrm{R}-\mathrm{C} \equiv \mathrm{CH}]+2 \mathrm{e}^{-}+2 \mathrm{H}^{+} \rightarrow \mathrm{Hg}+\left[\mathrm{R}-\mathrm{CH}=\mathrm{CH}_{2}\right]$ & 93 \\
$\mathrm{Hg}[2 \mathrm{R}-\mathrm{C}=\mathrm{N}-\mathrm{OH}]+4 \mathrm{e}^{-}+4 \mathrm{H}^{+} \rightarrow \mathrm{Hg}+\left[2 \mathrm{R}-\mathrm{CH}-\mathrm{NH}_{3}\right]$ & 102 \\
$\mathrm{Hg}\left[2 \mathrm{R}-\mathrm{C}=\mathrm{CH}_{2}\right]+2 \mathrm{e}^{-}+2 \mathrm{H}^{+} \rightarrow \mathrm{Hg}+\left[2 \mathrm{R}-\mathrm{CH}-\mathrm{CH}_{3}\right]$ & 102 \\
$\mathrm{Hg}[>\mathrm{C}=\mathrm{O}]+2 \mathrm{e}^{-}+2 \mathrm{H}^{+} \rightarrow \mathrm{Hg}+[>\mathrm{CH}-\mathrm{OH}]$ & 99 \\
\hline
\end{tabular}


Análises de formulações farmacêuticas por voltametria com HMDE são frequentes. Embora os excipientes possam ser eletroativos e os corantes presentes nas formulações possam adsorver no eletrodo de trabalho, em geral, estes apresentam pouca ou nenhuma interferência sobre o sinal analítico do analito. ${ }^{102-104}$ Técnicas de diluição da amostra juntamente com a aplicação de um potencial de pré-concentração quando são usadas técnicas de redissolução podem ser suficientes na minimização de possíveis interferências de matriz. Além disso, simples extrações dos fármacos empregando pequenas quantidades de solventes orgânicos podem ser empregadas. ${ }^{100,105}$

Ainda no âmbito de formulações, técnicas voltamétricas foram empregadas para a avaliação da presença de fármacos como adulterantes em composições fitoterápicas. Este estudo conduzido por Carvalho e colaboradores focou na presença de fármacos benzodiazepínicos (psicoativos) e anfepramona (anorexígeno) em medicamentos para emagrecimento teoricamente isentos de fármacos sintéticos. O eletrodo de trabalho HMDE e a técnica de redissolução por pulso diferencial foram empregados nesta investigação de adulterantes. Das 12 amostras analisadas, 4 apresentaram contaminação por benzodiazepínicos. As determinações foram realizadas com sucesso sem interferências de matriz, demonstrando a aplicabilidade do método desenvolvido. Quatro picos voltamétricos bem definidos correspondentes a quatro grupos de fármacos foram observados simultaneamente. ${ }^{54}$

Outra aplicação de interesse são as análises de amostras ambientais, e que pode ainda ser considerada um desafio analítico, principalmente devido à seletividade e sensibilidade..$^{22,93,95}$ Nunes et al. desenvolveram um método voltamétrico de screening para a determinação do benzodiazepínico (BDZ) alprazolam em águas superficiais. A técnica voltamétrica de redissolução catódica por pulso diferencial foi utilizada em determinações quantitativas. Amostras coletadas a jusante de uma estação de tratamento de água apresentaram uma concentração de $5 \mu \mathrm{g} \mathrm{L} \mathrm{L}^{-1}$.

Hormônios (semissintéticos e sintéticos) como o $17 \alpha$-etinilestradiol e o $17 \alpha$-metiltestosterona (MT) também foram determinados em amostras de águas naturais empregando o HMDE. ${ }^{93,95}$ Águas de rios e de tanques de criação de peixes foram analisadas por Miranda e colaboradores. As amostras coletadas em rios ficaram abaixo do LQ (10,78 $\left.\mu \mathrm{g} \mathrm{L}^{-1}\right)$, entretanto, das amostras coletadas em tanques de criação de peixes, as concentrações encontradas do hormônio MT variaram de $48 \pm 5,4$ a $142 \pm 15,5 \mu \mathrm{g} \mathrm{L}^{-1}$. Além de fármacos e hormônios, defensivos agrícolas também são contaminantes de ambientes naturais e vêm sendo estudados.

Nas últimas décadas, o uso desenfreado de produtos na agricultura fez despertar o interesse dos cientistas acerca da ocorrência e permanência de inseticidas, herbicidas, fungicidas, entre outros defensivos no solo e corpos aquáticos próximos. ${ }^{94,106-108}$ A presença destes contaminantes no ambiente pode ocasionar desequilíbrios ambientais além de efeitos nocivos, ainda não completamente elucidados, para a biota local e seres humanos. Assim como para os outros compostos orgânicos, técnicas cromatográficas são consideradas padrão para a determinação destas substâncias. Entretanto, a voltametria toma frente como técnica alternativa de análise destas substâncias em diversas matrizes. Trabalhos, inclusive brasileiros, empregaram o HMDE na determinação de pesticidas em distintas matrizes. Dos Santos et al. desenvolveram um método voltamétrico empregando a técnica de onda quadrada para a determinação do herbicida glifosato em amostras de águas naturais. Águas de rio e de um lago foram analisadas, sendo determinado o contaminante em uma das amostras analisadas do rio. ${ }^{108}$

Uma vez que defensivos agrícolas sejam utilizados para o controle de pragas na agricultura e consequentemente maior produção agrícola, estas substâncias nocivas estão inevitavelmente presentes nos alimentos e água que consumimos. Alimentos como vinho, frutas e grãos foram estudados a fim de determinar a contaminação e persistência de defensivos utilizados nos cultivares. ${ }^{109-113}$

Um método voltamétrico para a determinação do inseticida cifenotrina em formulações, vegetais e grãos foi desenvolvido por Kumar e Sreedhar. Pulso diferencial e o eletrodo HMDE foram empregados nas determinações voltamétricas em formulações, repolhos, tomates, trigo e arroz. Uma simples extração do analito empregando solventes orgânicos foi necessária, seguido pela determinação voltamétrica. Ótimas recuperações alcançadas demonstraram a eficácia do método. ${ }^{109}$ Galli e colaboradores, além de avaliarem a presença do herbicida pendimetalina em águas naturais e sedimentos, também realizaram a determinação deste composto em comida de bebê utilizando HMDE. Tal preocupação se justificou pelo fato de alguns ingredientes utilizados na elaboração do alimento, como maçã e mamão, serem culturas onde a aplicação de herbicidas é frequente. Embora altas concentrações de açucares e proteínas estivessem presentes nas amostras, determinações diretas, ou seja, sem pré-tratamentos, foram possíveis, e boas recuperações foram alcançadas pra amostras enriquecidas com o herbicida. ${ }^{114}$

A diversidade de compostos orgânicos determinados e matrizes analisadas mostra a enorme versatilidade do eletrodo de $\mathrm{Hg}$. Substâncias como fármacos podem ser determinados em composições, fluidos biológicos, amostras ambientais, entre outros de forma rápida, barata e simples. A aplicabilidade do HMDE se estende a compostos orgânicos das mais diferentes classes, como corantes, flavonoides, proteínas, pesticidas. ${ }^{115-119}$ De modo geral, todas as moléculas orgânicas que possuem sítios eletroativos podem ser determinadas empregando o eletrodo de $\mathrm{Hg}$. Moléculas não eletroativas na faixa de potencial de aplicação deste eletrodo, podem ser modificadas e assim tornar-se detectáveis por tal técnica. ${ }^{120,121} \mathrm{~A}$ Tabela 3 elenca diversas publicações realizadas nos últimos 10 anos com compostos orgânicos empregando HMDE como eletrodo de trabalho.

Na Tabela 3 nota-se a ampla aplicabilidade dos métodos voltamétricos na determinação de compostos orgânicos. Obviamente, as consideráveis vantagens supracitadas deste eletrodo, como o baixo custo operacional e de equipamento, simplicidade e versatilidade, foram decisivas para a escolha da técnica de análise por tantos pesquisadores.

\section{CONSIDERAÇÕES FINAIS}

A utilização do HMDE como eletrodo de trabalho vem ocorrendo a muitos anos devido suas inúmeras vantagens. A dispensabilidade ou simplicidade de pré-tratamentos de amostra reduz custos e aumenta a frequência analítica da técnica, além de evitar perdas do analito e contaminações das amostras. Baixos limites de detecção e sensibilidade tornam a voltametria com HMDE uma opção plausível para uma gama de finalidades. Os artigos apresentados nesta revisão exemplificam e ressaltam a diversidade de analitos e amostras que podem ser determinados e analisadas, respectivamente. Mesmo com o advento de novas técnicas analíticas, seja para compostos orgânicos ou não, a voltametria empregando o HMDE pode ter perdido um pouco de espaço, especialmente nos países mais desenvolvidos, mas ainda vem sendo empregada em diversos estudos nas áreas ambientais, farmacêuticas, forenses, entre outros. Em relação a toxicidade do eletrodo, vale ressaltar que volumes ínfimos de $\mathrm{Hg}$ são utilizados e todos os cuidados cabíveis podem ser tomados para evitar a volatilização do $\mathrm{Hg}$ durante as análises e na estocagem do resíduo gerado. Além disso, os resíduos de $\mathrm{Hg}$ gerados podem ser recuperados, purificados e reutilizados, não restando resíduos tóxicos descartáveis após as medições voltamétricas. Porém, assim como para outras técnicas analíticas, os pontos negativos devem ser considerados no momento da escolha da técnica. 
Tabela 3. Uso da voltametria e HMDE como eletrodo de trabalho na determinação de compostos orgânicos

\begin{tabular}{|c|c|c|c|c|c|c|}
\hline Analito & Classe / Função & Amostra & Método & LD & Faixa Linear & Ref \\
\hline acebutolol & $\begin{array}{l}\text { antiarrítmico e anti- } \\
\text { hipertensivo }\end{array}$ & $\begin{array}{c}\text { formulações } \\
\text { farmacêuticas, fluidos } \\
\text { biológicos }\end{array}$ & $\mathrm{CV}, \mathrm{SW}-\mathrm{SV}$ & $1,7 \times 10^{-7} \mathrm{~mol} \mathrm{~L}^{-1}$ & $5 \times 10^{-7}-6 \times 10^{-6} \mathrm{~mol} \mathrm{~L}^{-1}$ & 122 \\
\hline acibenzolar-S-metil & fungicida & $\begin{array}{c}\text { água de torneira, água } \\
\text { natural, solo }\end{array}$ & $\mathrm{CV}, \mathrm{SW}-\mathrm{SV}$ & $3,0 \times 10^{-9} \mathrm{~mol} \mathrm{~L}^{-1}$ & $1,0 \times 10^{-8}-6,0 \times 10^{-8} \mathrm{~mol} \mathrm{~L}^{-1}$ & 106 \\
\hline alprazolam & psicotrópico & água de rio & $\mathrm{CV}, \mathrm{DP}-\mathrm{SV}$ & $0,1 \mu \mathrm{g} \mathrm{L}^{-1}$ & $5,0-20 \mu \mathrm{g} \mathrm{L}^{-1}$ & 92 \\
\hline $\begin{array}{l}\text { albumina de soro } \\
\text { bovino }\end{array}$ & proteína & estudo eletroquímico & $\mathrm{CV}$ & NI & NI & 123 \\
\hline anfepramona & anoréxico & $\begin{array}{c}\text { estudo eletroquími- } \\
\text { co, formulações } \\
\text { farmacêuticas }\end{array}$ & $\begin{array}{l}\mathrm{CV}, \mathrm{AC} \\
\mathrm{DP}-\mathrm{CSV}\end{array}$ & $0,05-2,0 \mathrm{mg} \mathrm{L}^{-1}$ & $0,035-0,18 \mathrm{mg} \mathrm{L}^{-1}$ & 124 \\
\hline apo-metalotioneína & proteína & estudo eletroquímico & $\mathrm{DP}-\mathrm{SV}$ & $20 \mathrm{ng} \mathrm{mL}^{-1}$ & $0,015-12 \mathrm{n} \mathrm{mol} \mathrm{L}^{-1}$ & 125 \\
\hline $\begin{array}{l}\text { atrazina, ametrina, } \\
\text { simazina }\end{array}$ & herbicidas & estudo eletroquímico & SW & $3,7-7,5 \mu \mathrm{g} \mathrm{L}^{-1}$ & $1,0-10,0 \times 10^{-6} \mathrm{~mol} \mathrm{~L}^{-1}$ & 126 \\
\hline azinfos-etil & inseticida & $\begin{array}{l}\text { solo, água potável, } \\
\text { água residual }\end{array}$ & $\mathrm{CV}, \mathrm{DP}-\mathrm{SV}$ & $5,42 \times 10^{-9} \mathrm{~mol} \mathrm{~L}^{-1}$ & $1,28 \times 10^{-8}-4,39 \times 10^{-7} \mathrm{~mol} \mathrm{~L}^{-1}$ & 107 \\
\hline $\begin{array}{l}\text { benzodiazepínicos, } \\
\text { anfepramona }\end{array}$ & $\begin{array}{c}\text { psicotrópicos, } \\
\text { anorexígeno }\end{array}$ & $\begin{array}{l}\text { formulações fitoterá- } \\
\text { picas }\end{array}$ & DP - SV & NI & $1-30 \times 10^{-6} \mathrm{~mol} \mathrm{~L}^{-1}$ & 54 \\
\hline cefdinir & antibiótico & $\begin{array}{c}\text { formulações } \\
\text { farmacêuticas, urina }\end{array}$ & $\mathrm{CV}, \mathrm{SW}-\mathrm{SV}$ & $0,5 \times 10^{-9} \mathrm{~mol} \mathrm{~L}^{-1}$ & $1,88 \times 10^{-6}-12 \times 10^{-8} \mathrm{~mol} \mathrm{~L}^{-1}$ & 102 \\
\hline cefdinir & antibiótico & $\begin{array}{c}\text { formulações } \\
\text { farmacêuticas, soro }\end{array}$ & $\mathrm{CV}, \mathrm{SW}-\mathrm{SV}$ & $0,063 \mu \mathrm{mol} \mathrm{L}^{-1}$ & $0,25-40,0 \mu \mathrm{mol} \mathrm{L}^{-1}$ & 105 \\
\hline cefditoren pivoxil & antibiótico & $\begin{array}{c}\text { formulações } \\
\text { farmacêuticas, soro }\end{array}$ & $\mathrm{CV}, \mathrm{SW}-\mathrm{SV}$ & $0,1 \mu \mathrm{mol} \mathrm{L}^{-1}$ & $0,15-15,0 \mu \mathrm{mol} \mathrm{L}-1$ & 127 \\
\hline cefoperazona & antibiótico & $\begin{array}{c}\text { formulações } \\
\text { farmacêuticas, urina }\end{array}$ & $\mathrm{CV}, \mathrm{DP}, \mathrm{SW}-\mathrm{SV}$ & $\mathrm{NI}$ & $0,01-0,06 \mu \mathrm{g} \mathrm{mL} L^{-1}$ & 128 \\
\hline cifenotrina & inseticida & $\begin{array}{c}\text { formulações, vegetais, } \\
\text { grãos }\end{array}$ & $\mathrm{CV}, \mathrm{DP}-\mathrm{SV}$ & $1,9 \times 10^{-10} \mathrm{~mol} \mathrm{~L}^{-1}$ & $2 \times 10^{-9}-2 \times 10^{-5} \mathrm{~mol} \mathrm{~L}^{-1}$ & 109 \\
\hline ceftiofur & antibiótico & leite & SW - CSV & $1,86 \mathrm{ng} \mathrm{mL}^{-1}$ & $52,4-524 \mathrm{ng} \mathrm{mL}^{-1}$ & 23 \\
\hline ciprofloxacina & antibiótico & $\begin{array}{c}\text { forma pura, formula- } \\
\text { ções farmacêuticas, } \\
\text { urina } \\
\end{array}$ & CV, DP, SW - SV & $\mathrm{NI}$ & $0,05-0,15 \mu \mathrm{g} \mathrm{mL} L^{-1}$ & 103 \\
\hline $\begin{array}{l}\text { cloridrato de nortrip- } \\
\text { tilina }\end{array}$ & antidepressivo & $\begin{array}{l}\text { forma pura, formula- } \\
\text { ção farmacêutica }\end{array}$ & $\mathrm{CV}, \mathrm{SW}, \mathrm{DP}$ - SV & $0,92 \mathrm{ng} \mathrm{mL}^{-1}$ & $2 \times 10^{-6}-1 \times 10^{-7} \mathrm{~mol} \mathrm{~L}^{-1}$ & 129 \\
\hline clorotetraciclina & antibiótico & $\begin{array}{c}\text { formulações } \\
\text { farmacêuticas, fluidos } \\
\text { biológicos }\end{array}$ & DP, SW - SV & $7,2 \times 10^{-9} \mathrm{~mol} \mathrm{~L}^{-1}$ & $1 \times 10^{-7}-1,4 \times 10^{-6} \mathrm{~mol} \mathrm{~L}^{-1}$ & 130 \\
\hline clorpirifós & inseticida & vinho & $\mathrm{CV}, \mathrm{DP}-\mathrm{SV}$ & $0,14 \mathrm{ng} \mathrm{mL}^{-1}$ & $0,2-1,2 \mathrm{ng} \mathrm{mL}^{-1}$ & 110 \\
\hline creatina & aminoácido & $\begin{array}{l}\text { água, soro de } \\
\text { sangue, formulações } \\
\text { farmacêuticas }\end{array}$ & DP - SV & $0,11 \mathrm{ng} \mathrm{mL}^{-1}$ & $0,11 \mathrm{ng} \mathrm{mL}^{-1}-23,00 \mu \mathrm{g} \mathrm{mL}^{-1}$ & 131 \\
\hline danofloxacin & antibiótico & $\begin{array}{c}\text { formulações } \\
\text { farmacêuticas }\end{array}$ & $\mathrm{CV}, \mathrm{DP}-\mathrm{SV}$ & $5,5 \times 10^{-8} \mathrm{~mol} \mathrm{~L}^{-1}$ & $4 \times 10^{-8}-2,4 \times 10^{-7} \mathrm{~mol} \mathrm{~L}^{-1}$ & 132 \\
\hline diazinon & inseticida & formulações & $\mathrm{CV}, \mathrm{SW}-\mathrm{SV}$ & $1,1 \times 10^{-8} \mathrm{~mol} \mathrm{~L}^{-1}$ & $4,0 \times 10^{-8}-3,9 \times 10^{-7} \mathrm{~mol} \mathrm{~L}^{-1}$ & 133 \\
\hline dienos conjugados & substância orgânica & gasolina de pirólise & DP & NI & NI & 134 \\
\hline ebastina & anti-histamínicos & $\begin{array}{c}\text { composições } \\
\text { farmacêuticas }\end{array}$ & CV, DP - SV & $0,48 \times 10^{-8} \mathrm{~mol} \mathrm{~L}^{-1}$ & $2,0 \times 10^{-8}-1,0 \times 10^{-7} \mathrm{~mol} \mathrm{~L}^{-1}$ & 135 \\
\hline enrofloxacin & antibiótico & $\begin{array}{l}\text { plasma, formulações } \\
\text { farmacêuticas }\end{array}$ & SW - SV & $0,33 \mathrm{n} \mathrm{mol} \mathrm{L}{ }^{-1}$ & $10,0-80,0 \mathrm{n} \mathrm{mol} \mathrm{L}{ }^{-1}$ & 121 \\
\hline entacapone & $\begin{array}{l}\text { tratamento de par- } \\
\text { kinson }\end{array}$ & $\begin{array}{c}\text { formulações } \\
\text { farmacêuticas }\end{array}$ & CV, DP, SW - SV & $0,13 \mathrm{ng} \mathrm{mL}^{-1}$ & $5 \times 10^{-4}-1,8 \times 10^{-5} \mathrm{~mol} \mathrm{~L}^{-1}$ & 136 \\
\hline $\operatorname{eosin}-Y$ & corante & $\begin{array}{c}\text { produto comercial } \\
\text { (varnish), água } \\
\text { marinha }\end{array}$ & $\mathrm{CV}, \mathrm{SW}-\mathrm{SV}$ & $8,73 \times 10^{-10} \mathrm{~mol} \mathrm{~L}^{-1}$ & $5,0 \times 10^{-8}-5,0 \times 10^{-7} \mathrm{~mol} \mathrm{~L}^{-1}$ & 137 \\
\hline febuxostate & $\begin{array}{l}\text { inibidor de xantina- } \\
\text { oxidase }\end{array}$ & $\begin{array}{c}\text { formulações } \\
\text { farmacêuticas, plasma } \\
\text { sanguíneo }\end{array}$ & PD - SV & $6,93 \mathrm{ng} \mathrm{mL}^{-1}$ & $12,5-200 \mathrm{ng} \mathrm{mL}^{-1}$ & 138 \\
\hline fenitrothion, malathion & inseticidas & estudo eletroquímico & DP - SV & $\mathrm{NI}$ & $\mathrm{NI}$ & 139 \\
\hline fenoxaprop-p-ethyl & herbicida & $\begin{array}{l}\text { água de torneira, água } \\
\text { de rios, plantas }\end{array}$ & $\mathrm{CV}, \mathrm{SW}-\mathrm{SV}$ & $0,07 \mu \mathrm{mol} \mathrm{L}^{-1}$ & $0,30-15 \mu \mathrm{mol} \mathrm{L}^{-1}$ & 140 \\
\hline FTT1103 & proteína & estudo eletroquímico & $\mathrm{AC}-\mathrm{SV}$ & $45 \mathrm{n} \mathrm{mol} \mathrm{L}^{-1}$ & $1-5 \mu \mathrm{mol} \mathrm{L}^{-1}$ & 115 \\
\hline $17 \alpha$-etinilestradiol & hormônio & água natural & $\mathrm{CV}, \mathrm{DP}-\mathrm{SV}$ & $0,49 \mu \mathrm{g} \mathrm{L} \mathrm{L}^{-1}$ & $2,0-96,0 \mu \mathrm{g} \mathrm{L}^{-1}$ & 93 \\
\hline
\end{tabular}


Tabela 3. Uso da voltametria e HMDE como eletrodo de trabalho na determinação de compostos orgânicos (cont.)

\begin{tabular}{|c|c|c|c|c|c|c|}
\hline Analito & Classe / Função & Amostra & Método & $\mathrm{LD}$ & Faixa Linear & Ref. \\
\hline gatifloxacin & antibiótico & $\begin{array}{c}\text { formulações } \\
\text { farmacêuticas, sangue } \\
\text { humano }\end{array}$ & SW - SV & $1,5 \mathrm{n} \mathrm{mol} \mathrm{L}^{-1}$ & $5,0 \times 10^{-9}-3,0 \times 10^{-7} \mathrm{~mol} \mathrm{~L}^{-1}$ & 141 \\
\hline gefitinib & antitumoral & $\begin{array}{c}\text { formulações } \\
\text { farmacêuticas, urina }\end{array}$ & $\mathrm{CV}$ & $1.2 \times 10^{8} \mathrm{~mol} \mathrm{~L}^{-1}$ & $1,0 \times 10^{4}-1,0 \times 10^{8} \mathrm{~mol} \mathrm{~L}^{-1}$ & 142 \\
\hline glifosato & herbicida & águas naturais & $\mathrm{CV}, \mathrm{SW}-\mathrm{SV}$ & $22,04 \mu \mathrm{g} \mathrm{L} \mathrm{L}^{-1}$ & $3,93 \times 10^{-7}-3,52 \times 10 \mathrm{~mol} \mathrm{~L}^{-1}$ & 108 \\
\hline granisetron & antiemético & $\begin{array}{c}\text { formulações } \\
\text { farmacêuticas, } \\
\text { biológica } \\
\end{array}$ & $\mathrm{CV}, \mathrm{SW}-\mathrm{SV}$ & $3,63 \mathrm{ng} \mathrm{mL}^{-1}$ & $0-200 \mathrm{ng} \mathrm{mL}^{-1}$ & 100 \\
\hline haloperidol & psicotrópico & estudo eletroquímico & $\mathrm{CV}, \mathrm{SW}-\mathrm{SV}$ & $4,75 \times 10^{-9} \mathrm{~mol} \mathrm{~L}^{-1}$ & $2,49 \times 10^{-7}-3,38 \times 10^{-6} \mathrm{~mol} \mathrm{~L}^{-1}$ & 143 \\
\hline 3-hydroxyflavone & flavonoide & $\begin{array}{l}\text { a granel, fluidos } \\
\text { biológicos }\end{array}$ & CV, SW - SV & $4,4-7,54 \mathrm{n} \mathrm{mol} \mathrm{L}-1$ & $0,019-0,654 \mu \mathrm{mol} \mathrm{L}-1$ & 144 \\
\hline irbesartan & anti-hipertensivo & $\begin{array}{c}\text { formulações } \\
\text { farmacêuticas, soro } \\
\text { sanguíneo }\end{array}$ & CV, SW - SV & $9,0 \times 10^{-10} \mathrm{~mol} \mathrm{~L}^{-1}$ & $3,0 \times 10^{-9}-5,0 \times 10^{-7} \mathrm{~mol} \mathrm{~L}^{-1}$ & 145 \\
\hline $\begin{array}{l}\text { lemofloxacina, clori- } \\
\text { drato de esparfloxacina, } \\
\text { gatifloxacina, moxi- } \\
\text { floxacina }\end{array}$ & antibióticos & $\begin{array}{l}\text { soro sanguíneo, } \\
\text { urina, formulações } \\
\text { farmacêuticas }\end{array}$ & CV, DP - CSV & $2 \times 10^{-8} \mathrm{~mol} \mathrm{~L}^{-1}$ & $10^{-7}-10^{-8} \mathrm{~mol} \mathrm{~L}^{-1}$ & 146 \\
\hline levobunolol & anti-glaucoma & $\begin{array}{c}\text { formulações } \\
\text { farmacêuticas, soro }\end{array}$ & $\mathrm{CV}, \mathrm{SW}-\mathrm{SV}$ & $3,0 \times 10^{-11} \mathrm{~mol} \mathrm{~L}^{-1}$ & $1,0 \times 10^{-10}-3,0 \times 10^{-8} \mathrm{~mol} \mathrm{~L}^{-1}$ & 104 \\
\hline losartan & anti-hipertensivo & $\begin{array}{l}\text { forma pura, formula- } \\
\text { ções farmacêuticas }\end{array}$ & $\begin{array}{c}\mathrm{CV}, \mathrm{DC}, \mathrm{DP}, \mathrm{SW}, \\
\mathrm{AC}\end{array}$ & 0,12 a $0,15 \mu \mathrm{g} \mathrm{mL}-1$ & $0,16-1,2 \mu \mathrm{g} \mathrm{mL} L^{-1}$ & 147 \\
\hline mesotriona & herbicida & solo & $\mathrm{CV}, \mathrm{DP}$ & $50 \mathrm{n} \mathrm{mol} \mathrm{L}^{-1}$ & $0,10-10,0 \mu \mathrm{mol} \mathrm{L}^{-1}$ & 148 \\
\hline $\mathrm{MtSH}, \mathrm{EtSH}, \mathrm{PrSH}$ & compostos de enxofre & fluidos hidrotermais & DP - CSV & $1,1 \mu \mathrm{mol} \mathrm{L}-1$ & 2,6 a $10 \mu \mathrm{mol} \mathrm{L}^{-1}$ & 71 \\
\hline methidathion & inseticida, acaricida & estudo eletroquímico & $\mathrm{CV}, \mathrm{SW}-\mathrm{SV}$ & $98,5 \mathrm{n} \mathrm{mol} \mathrm{L}^{-1}$ & $0,2-3,85 \mu \mathrm{mol} \mathrm{L}^{-1}$ & 149 \\
\hline $17 \alpha$ - metiltestosterona & hormônio & água natural & CV, SW - SV & $1,0 \times 10^{-8} \mathrm{~mol} \mathrm{~L}^{-1}$ & $4,6 \times 10^{-8}-4,2 \times 10^{-7} \mathrm{~mol} \mathrm{~L}^{-1}$ & 95 \\
\hline $17 \alpha$ - metiltestosterona & hormônio & água natural & SW - SV & $3,07 \mu \mathrm{g} \mathrm{L}^{-1}$ & $4,6 \times 10^{-8}-4,2 \times 10^{-7} \mathrm{~mol} \mathrm{~L}^{-1}$ & 150 \\
\hline metribuzina & herbicida & $\begin{array}{c}\text { água de torneira e } \\
\text { natural }\end{array}$ & $\begin{array}{c}\mathrm{CV} \text {, linear, DP } \\
-\mathrm{SV}\end{array}$ & $2,7 \times 10^{-9} \mathrm{~mol} \mathrm{~L}^{-1}$ & $1 \times 10^{-6}-3,5 \times 10^{-5} \mathrm{~mol} \mathrm{~L}^{-1}$ & 11 \\
\hline montelukast & $\begin{array}{c}\text { tratamento de doenças } \\
\text { respiratórias }\end{array}$ & $\begin{array}{l}\text { formulações, urina, } \\
\text { plasma }\end{array}$ & $\mathrm{CV}, \mathrm{SW}-\mathrm{SV}$ & $4 \times 10^{-9} \mathrm{~mol} \mathrm{~L}^{-1}$ & $5 \times 10^{-8}-1 \times 10^{-6} \mathrm{~mol} \mathrm{~L}^{-1}$ & 151 \\
\hline murexida & corante indicador & estudo eletroquímico & $\mathrm{CV}$ & $\mathrm{NI}$ & NI & 29 \\
\hline nifedipina & anti-hipertensivo & $\begin{array}{c}\text { formulações } \\
\text { farmacêuticas }\end{array}$ & CV, DP -SV & NI & $1 \times 10^{-8}-1 \times 10^{-7} \mathrm{~mol} \mathrm{~L}^{-1}$ & 152 \\
\hline nimodipine & anti-hipertensivo & $\begin{array}{c}\text { formulações } \\
\text { farmacêuticas }\end{array}$ & $\mathrm{CV}, \mathrm{SW}, \mathrm{PD}-\mathrm{SV}$ & $7,11 \mathrm{ng} \mathrm{mL} L^{-1}$ & $2 \times 10^{-5}-2 \times 10^{-6} \mathrm{~mol} \mathrm{~L}^{-1}$ & 153 \\
\hline nitazoxanida & antiparasitário & $\begin{array}{c}\text { formulações } \\
\text { farmacêuticas, soro }\end{array}$ & $\mathrm{CV}, \mathrm{SW}-\mathrm{SV}$ & $\begin{array}{c}3,0 \times 10^{-11}-2,4 \times 10^{-10} \\
\mathrm{~mol} \mathrm{~L}^{-1}\end{array}$ & $1 \times 10^{-9}-2 \times 10^{-7} \mathrm{~mol} \mathrm{~L}^{-1}$ & 97 \\
\hline nitrotal-isopropil & fungicida & águas naturais & $\mathrm{CV}, \mathrm{SW}-\mathrm{SV}$ & $3,46 \times 10^{-8} \mathrm{~mol} \mathrm{~L}^{-1}$ & $2,0 \times 10^{-7}-2,0 \times 10^{-6} \mathrm{~mol} \mathrm{~L}^{-1}$ & 154 \\
\hline novobiocina & antibiótico & estudo eletroquímico & SW & NI & NI & 155 \\
\hline ofloxacin & antibiótico & $\begin{array}{l}\text { comprimidos, fluidos } \\
\text { biológicos }\end{array}$ & $\mathrm{CV}, \mathrm{SW}-\mathrm{SV}$ & $1,1 \times 10^{-8} \mathrm{~mol} \mathrm{~L}^{-1}$ & $5 \times 10^{-7}-1,7 \times 10^{-6} \mathrm{~mol} \mathrm{~L}^{-1}$ & 98 \\
\hline $\begin{array}{l}\text { olmesartana me- } \\
\text { doxomila }\end{array}$ & anti-hipertensivo & $\begin{array}{c}\text { formulações } \\
\text { farmacêuticas }\end{array}$ & CV, DP - SV & $1,53 \times 10^{-7} \mathrm{~mol} \mathrm{~L}^{-1}$ & $4,7 \times 10^{-8}-8,3 \times 10^{-6} \mathrm{~mol} \mathrm{~L}^{-1}$ & 156 \\
\hline olopatadina & antialérgico & $\begin{array}{c}\text { formulações } \\
\text { farmacêuticas }\end{array}$ & $\mathrm{CV}, \mathrm{DP}-\mathrm{SV}$ & $1,92 \mathrm{ng} \mathrm{mL}^{-1}$ & $1,0 \times 10^{-8}-4,0 \times 10^{-7} \mathrm{~mol} \mathrm{~L}^{-1}$ & 157 \\
\hline oxyfluorfen & herbicida & formulações agrícolas & $\mathrm{CV}, \mathrm{DP}$ & NI & $1,0 \times 10^{-5}-3,15 \times 10^{-7} \mathrm{~mol} \mathrm{~L}^{-1}$ & 158 \\
\hline pendimetalina & herbicida & $\begin{array}{l}\text { água, sedimentos, } \\
\text { comida de bebê }\end{array}$ & $\mathrm{CV}, \mathrm{SW}-\mathrm{SV}$ & $2,77 \times 10^{-8} \mathrm{~mol} \mathrm{~L}^{-1}$ & $5,0 \times 10^{-7}-4,5 \times 10^{-6} \mathrm{~mol} \mathrm{~L}^{-1}$ & 114 \\
\hline pindone & rodenticida & $\begin{array}{l}\text { composições agríco- } \\
\text { las, grãos }\end{array}$ & $\mathrm{CV}, \mathrm{DP}-\mathrm{SV}$ & $2,0 \mathrm{n} \mathrm{mol} \mathrm{L}^{-1}$ & $3,0 \times 10^{-9}-4,0 \times 10^{-7} \mathrm{~mol} \mathrm{~L}^{-1}$ & 116 \\
\hline POP's & pesticidas & $\begin{array}{l}\text { água marinha, } \\
\text { alimentos }\end{array}$ & $\begin{array}{l}\text { CV, Linear, } \\
\text { DP - SV }\end{array}$ & $\begin{array}{c}6,18 \times 10^{-12}-4,34 \times \\
10^{-12} \mathrm{~mol} \mathrm{~L}^{-1}\end{array}$ & $\begin{array}{l}7,4 \times 10^{-9}-1,4 \times 10^{-7} \mathrm{~mol} \mathrm{~L}^{-1} \\
2,7 \times 10^{-9}-1,6 \times 10^{-8} \mathrm{~mol} \mathrm{~L}^{-1}\end{array}$ & 111 \\
\hline PPB & líquido iônico & $\begin{array}{l}\text { detergentes, água } \\
\text { marinha }\end{array}$ & $\mathrm{CV}-\mathrm{SV}$ & $6,4 \times 10^{-7} \mathrm{~mol} \mathrm{~L}^{-1}$ & $1,0 \times 10^{-5}-5,0 \times 10^{-4} \mathrm{~mol} \mathrm{~L}^{-1}$ & 159 \\
\hline quinina & $\begin{array}{l}\text { analgésico, antitér- } \\
\text { mico }\end{array}$ & $\begin{array}{l}\text { plantas, formulações } \\
\text { farmacêuticas, estu- } \\
\text { dos eletroquímicos } \\
\end{array}$ & $\mathrm{CV}, \mathrm{SW}-\mathrm{SV}$ & $0,132 \mathrm{ng} \mathrm{mL}^{-1}$ & $30,0-230,0 \mathrm{ng} \mathrm{mL} \mathrm{m}^{-1}$ & 160 \\
\hline
\end{tabular}


Tabela 3. Uso da voltametria e HMDE como eletrodo de trabalho na determinação de compostos orgânicos (cont.)

\begin{tabular}{|c|c|c|c|c|c|c|}
\hline Analito & Classe / Função & Amostra & Método & $\mathrm{LD}$ & Faixa Linear & Ref \\
\hline quinometionato & acaricida & frutas & $\mathrm{CV}, \mathrm{DP}-\mathrm{SV}$ & $0,098 \mathrm{ng} \mathrm{mL}^{-1}$ & $0,5-5,0 \mathrm{ng} \mathrm{mL}^{-1}$ & 112 \\
\hline quitosana & polissacarídeo & estudo eletroquímico & $\mathrm{CV}, \mathrm{SW}-\mathrm{SV}$ & $10 \mathrm{ng} \mathrm{mL}^{-1}$ & $0,7-2,5 \mu \mathrm{g} \mathrm{mL}^{-1}$ & 161 \\
\hline RB5 & corante & $\begin{array}{l}\text { cultura de fungos (es- } \\
\text { tudos de degradaçãao } \\
\text { do corante) }\end{array}$ & DP - SV & NI & NI & 162 \\
\hline RB5 & corante & água de descarte & $\mathrm{CV}, \mathrm{DP}-\mathrm{SV}$ & $1,3 \times 10^{-8} \mathrm{~mol} \mathrm{~L}^{-1}$ & $2,5 \times 10^{-7}-2,0 \times 10^{-6} \mathrm{~mol} \mathrm{~L}^{-1}$ & 117 \\
\hline repaglinida & antidiabético & $\begin{array}{l}\text { formulações } \\
\text { farmacêuticas }\end{array}$ & $\begin{array}{c}\text { CV, DC, DP, SW } \\
- \text { SV }\end{array}$ & $0,053 \mathrm{mg} \mathrm{L}^{-1}$ & $0,453-10,86 \mathrm{mg} \mathrm{L}^{-1}$ & 163 \\
\hline$\underline{\text { resazurina }}$ & corante & estudo eletroquímico & $\mathrm{CV}, \mathrm{SW}-\mathrm{SV}$ & $\mathrm{NI}$ & NI & 5 \\
\hline riboflavina & vitamina B2 & Estudo eletroquímico & $\mathrm{CV}, \mathrm{DP}-\mathrm{SV}$ & $7 \mu \mathrm{g} \mathrm{L}^{-1}$ & $17 \mu \mathrm{g} \mathrm{L}^{-1}-2 \mathrm{mg} \mathrm{L}^{-1}$ & 164 \\
\hline rosiglitazona & antidiabético & urina, plasma & $\mathrm{CV}, \mathrm{SW}-\mathrm{SV}$ & $3,2 \times 10^{-11} \mathrm{~mol} \mathrm{~L}^{-1}$ & $5 \times 10^{-8}-8 \times 10^{-7} \mathrm{~mol} \mathrm{~L}^{-1}$ & 165 \\
\hline rosuvastatina & redução de gordura & $\begin{array}{l}\text { composições } \\
\text { farmacêuticas }\end{array}$ & CV, DP - SV & $0,112 \mathrm{ng} \mathrm{mL}^{-1}$ & $0,96-192,61 \mathrm{ng} \mathrm{mL}^{-1}$ & 96 \\
\hline rutina & flavonoide & soja & $\mathrm{CV}, \mathrm{DP}-\mathrm{SV}$ & $7 \times 10^{-9} \mathrm{~mol} \mathrm{~L}^{-1}$ & $2 \times 10^{-7}-1,4 \times 10^{-6} \mathrm{~mol} \mathrm{~L}^{-1}$ & 120 \\
\hline $\begin{array}{l}\text { Triton X-100, ácido } \\
\text { húmico }\end{array}$ & $\begin{array}{c}\text { substâncias ativas de } \\
\text { superfície }\end{array}$ & águas naturais & $\mathrm{AC}$ & NI & $0,02-0,25 \mathrm{mg} \mathrm{L}^{-1}$ & 166 \\
\hline SAS & $\begin{array}{c}\text { substâncias ativas de } \\
\text { superfície }\end{array}$ & ar & $\mathrm{AC}$ & $0,02 \mathrm{mg} \mathrm{dm}^{-3}$ & $0,02-1 \mathrm{mg} \mathrm{dm}^{-3}$ & 167 \\
\hline sibutramina & anti-obesidade & $\begin{array}{l}\text { bebidas, formulações } \\
\text { farmacêuticas }\end{array}$ & $\mathrm{CV}, \mathrm{SW}$ & $0,4 \mathrm{mg} \mathrm{L}^{-1}$ & $1,4-33,3 \mathrm{mg} \mathrm{L}^{-1}$ & 119 \\
\hline sudan I & corante & bebidas & SW - SV & $1,5 \mu \mathrm{g} \mathrm{L}^{-1}$ & $0,5-27,2 \mu \mathrm{g} \mathrm{L}{ }^{-1}$ & 168 \\
\hline tacrolimo & imunodepressor & $\begin{array}{l}\text { formulações } \\
\text { farmacêuticas }\end{array}$ & $\mathrm{CV}, \mathrm{DP}-\mathrm{SV}$ & $0,11 \mathrm{ng} \mathrm{mL}^{-1}$ & $1-60 \mathrm{ng} \mathrm{mL}^{-1}$ & 169 \\
\hline $\begin{array}{l}\text { tartrazina, amarelo } \\
\text { crepusculo }\end{array}$ & corantes & $\begin{array}{c}\text { gelatina, pó para } \\
\text { refrigerante }\end{array}$ & SW - SV & 3,$3 ; 1,6 \mu \mathrm{g} \mathrm{L}^{-1}$ & $6,6-300 \mu \mathrm{g} \mathrm{L}-1 ; 3,3-160 \mu \mathrm{g} \mathrm{L}^{-1}$ & 170 \\
\hline timolol & anti-hipertensivo & $\begin{array}{l}\text { compostos farmacêu- } \\
\text { ticos, fluidos biológi- } \\
\cos \\
\end{array}$ & $\mathrm{CV}, \mathrm{DP}-\mathrm{SV}$ & $1,26 \mathrm{n} \mathrm{mol} \mathrm{L}^{-1}$ & $1 \times 10^{-7}-1,5 \times 10^{-6} \mathrm{~mol} \mathrm{~L}^{-1}$ & 171 \\
\hline tolmetina & anti-inflamatório & $\begin{array}{l}\text { forma livre, formula- } \\
\text { ções farmacêuticas, } \\
\text { soro }\end{array}$ & $\mathrm{CV}, \mathrm{SW}-\mathrm{SV}$ & $6 \times 10^{-10} \mathrm{~mol} \mathrm{~L}^{-1}$ & $2 \times 10^{-9}-5 \times 10^{-8} \mathrm{~mol} \mathrm{~L}^{-1}$ & 99 \\
\hline trazodona & anti-depressivo & $\begin{array}{c}\text { formulações } \\
\text { farmacêuticas, soro }\end{array}$ & $\mathrm{CV}, \mathrm{SW}-\mathrm{SV}$ & $4,32 \mathrm{n} \mathrm{mol} \mathrm{L}^{-1}$ & $8,0-610,0 \mathrm{n} \mathrm{mol} \mathrm{L}-1$ & 172 \\
\hline triasufulron & herbicida & $\begin{array}{c}\text { preparações, águas } \\
\text { naturais }\end{array}$ & $\mathrm{CV}, \mathrm{DC}, \mathrm{DP}-\mathrm{SV}$ & $2,7 \mathrm{n} \mathrm{mol} \mathrm{L}^{-1}$ & $5 \times 10^{-9}-5 \times 10^{-6} \mathrm{~mol} \mathrm{~L}^{-1}$ & 173 \\
\hline vlone & rodenticida & água & CV, DP - SV & $1,0 \times 10^{-5} \mathrm{~mol} \mathrm{~L}^{-1}$ & $1,0 \times 10^{-3}-1,0 \times 10^{-5} \mathrm{~mol} \mathrm{~L}^{-1}$ & 118 \\
\hline valsartan & anti-hipertensivo & $\begin{array}{l}\text { a granel, formulações } \\
\text { farmacêuticas }\end{array}$ & $\begin{array}{c}\text { CV, AC, DC, DP, } \\
\text { SW - SV }\end{array}$ & $0,12 \mu \mathrm{g} \mathrm{mL}^{-1}$ & $0,16-1,2 \mu \mathrm{g} \mathrm{mL}-1$ & 174 \\
\hline zalcitabina & antiviral sistêmico & $\begin{array}{c}\text { formulações } \\
\text { farmacêuticas }\end{array}$ & $\mathrm{CV}, \mathrm{DP}-\mathrm{SV}$ & $2,08 \mathrm{mg} \mathrm{L}^{-1}$ & $10,0-28,0 \mathrm{mg} \mathrm{L}^{-1}$ & 175 \\
\hline zanozar & agente quimioterápico & $\begin{array}{c}\text { formulações } \\
\text { farmacêuticas, urina }\end{array}$ & $\mathrm{CV}, \mathrm{DC}, \mathrm{DP}-\mathrm{SV}$ & $7,42 \times 10^{-8} \mathrm{~mol} \mathrm{~L}^{-1}$ & $1,0 \times 10^{-7}-1,0 \times 10^{-3} \mathrm{~mol} \mathrm{~L}^{-1}$ & 176 \\
\hline ziram & fungicida & arroz & $\mathrm{DP}-\mathrm{SV}$ & $1 \mu \mathrm{g} \mathrm{L}^{-1}$ & até $305 \mu \mathrm{g} \mathrm{L}^{-1}$ & 113 \\
\hline zolinza (vorinostat) & $\begin{array}{l}\text { inibidor de histona } \\
\text { deacetilases (trata- } \\
\text { mento de câncer) }\end{array}$ & $\begin{array}{c}\text { formulações } \\
\text { farmacêuticas, urina }\end{array}$ & $\mathrm{CV}, \mathrm{DP}$ & $0,98 \times 10^{-9} \mathrm{~mol} \mathrm{~L}^{-1}$ & $1 \times 10^{-5}-1 \times 10^{-9} \mathrm{~mol} \mathrm{~L}^{-1}$ & 177 \\
\hline
\end{tabular}

$\overline{\mathrm{AC}}=$ corrente alternada $\mathrm{CV}=$ voltametria cíclica; $\mathrm{DC}=$ corrente direta; $\mathrm{DP}=$ pulso diferencial; $\mathrm{NI}=$ não informado; $\mathrm{SV}=$ voltametria de redissolução; $\mathrm{SW}=$ onda quadrada.

\section{AGRADECIMENTOS}

Capes, CNPq, Fundação Araucária - Fundação Parque Tecnológico Itaipu e Prof. Dr. Fauze J. Anaissi pelo incentivo à publicação deste tema.

\section{REFERÊNCIAS}

1. Pacheco, W. F.; Semaan, F. S.; de Almeida, V. G. K.; Ritta, A. G. S. L.; Aucélio, Q.; Rev. Virtual Quim. 2013, 5, 516.

2. Bockris, J. O.; Reddy, A. K. N.; Gamboa-Aldeco, M.; Modern
Electrochemistry: Fundamentals of Electrodics, $2^{\text {nd }}$ ed., Springer, 2000.

3. Shukla, A. K.; Kumar, T. P.; Electrochem. Soc. 2008, 307483, 31.

4. Bagotsky, V. S.; Fundamentals of Electrochemistry, $2^{\text {nd }}$ ed., John Wiley \& Sons, Inc.: Hoboken, 2005.

5. Arslan, E. Y.; Çakir, S.; South African J. Chem. 2010, 63, 152.

6. EPA/625/R-94/004; Hanbook: Recycling and Reuse of Material Found on Superfund Sites 1994.

7. Volke, J.; J. Eletroanalytical Chem. 1983, 155, 7.

8. Bard, A. J.; Faulkner, L. R.; Electrochemical Methods: Fundamentals and Applications, $2^{\text {nd }}$ ed., Wiley, 2001.

9. Zuman, P.; Microchem. J. 2002, 72, 241 . 
10. Srikanth, P.; Somasekhar, S. A.; Kanthi, G. K.; Babu, K. R.; International Journal of Environment, Ecology, Family and Urban Studies 2013, 3, 127.

11. Janíková, L.; Šelešovská, R.; Rogozinská, M.; Tomášková, M.; Chýlková, J.; Monatsh. Chem. 2016, 147, 219.

12. de Carvalho, L. M.; do Nascimento, P. C.; Koschinsky, A.; Bau, M.; Stefanello, R. F.; Spengler, C.; Bohrer, D.; Jost, C.; Electroanalysis 2007, 19, 1719.

13. de Souza, D.; Melo, L. C.; Correia, A. N.; de Lima-Neto, P.; FatibelloFilho, O.; Mascaro, L. H.; Quim. Nova 2011, 34, 487.

14. Cao, L.; Jia, J.; Wang, Z.; Electrochim. Acta 2008, 53, 2177.

15. de Figueiredo-Filho, L. C. S.; Baccarin, M.; Janegitz, B. C.; FatibelloFilho, O.; Sens. Actuators, B 2017, 240, 749.

16. Jain, R.; Yadav, R. K.; J. Pharm. Anal. 2012, 2, 123.

17. Santos, M. C. G.; Tarley, C. R. T.; Dall'Antonia, L. H.; Sartori, E. R.; Sens. Actuators, B 2013, 188, 263.

18. Zidaric, T.; Jovanovski, V.; Menart, E.; Zorko, M.; Kolar, M.; Veber, M.; Hocevar, S. B.; Sens. Actuators, B 2017, 245, 720.

19. Romih, T.; Hočevar, S. B.; Jemec, A.; Drobne, D.; Electrochim. Acta 2016, 188, 393.

20. Grabarczyk, M.; Wasag, J.; Talanta 2015, 144, 1091.

21. Kong, D.; Kong, W.; Khan, Z. U. H.; Wan, P.; Chen, Y.; Yang, M.; Fuel 2016, 182, 266.

22. Gerent, G. G.; Spinelli, A.; J. Hazard. Mater. 2016, 308, 157.

23. Barbosa, A. M. J.; de Araújo, T. A.; Trindade, M. A. G.; Ferreira, V. S.; J. Appl. Electrochem. 2011, 41, 249.

24. Yang, M.; Wang, S.; Zhang, L.; Wu, Q.; Wang, F.; Hui, M.; Yang, H.; Hao, J.; J. Geochem. Explor. 2016, 170, 72.

25. Black, P.; Richard, M.; Rossin, R.; Telmer, K.; Environ. Res. 2017, 152, 462.

26. Wasserman, J.; Hacon, S.; Mundo e Vida 2001, 2, 46.

27. dos Anjos, V. E.; Abate, G.; Tadeu, G. M.; Quim. Nova 2010, 33, 1307

28. Tonietto, A. E.; Grassi, M. T.; Quim. Nova 2012, 35, 170

29. Mohran, H. S.; Am. J. Appl. Sci. 2009, 6, 964.

30. da Silveira, G. D.; Hoinacki, C. K.; Bueno Goularte, R.; do Nascimento, P. C.; Bohrer, D.; Cravo, M.; Leite, L. F. M.; de Carvalho, L. M.; Fuel 2017, 202, 206.

31. Repula, C. M. M.; Quináia, S. P.; de Campos, B. K.; Ganzarolli, E. M.; Lopes, M. C.; Bull. Environ. Contam. Toxicol. 2012, 88, 84.

32. Repula, C. M. M.; de Campos, B. K.; Ganzarolli, E. M.; Lopes, M. C.; Quináia, S. P.; Quim. Nova 2012, 35, 905.

33. Arancibia, V.; Zúñiga, M.; ZÚñiga, M. C.; Segura, R.; Esteban, M.; $J$. Braz. Chem. Soc. 2010, 21, 255.

34. Junsomboon, J.; Sooksamiti, P.; Grudpan, K.; Lapanantnoppakhun, S.; Thavornyuthikarn, P.; Jakmunee, J.; Chiang Mai J. Sci. 2009, 36, 369.

35. Locatelli, C.; Melucci, D.; Cent. Eur. J. Chem. 2013, 11, 790.

36. Chooto, P.; Wararattananurak, P.; Kangkamano, T.; Innuphat, C.; Sirinawin, W.; ScienceAsia 2015, 41, 187.

37. Ba-Shami, R. M.; Gazzaz, H.; Bashammakh, A. S.; Al-Sibaai, A. A.; El-Shahawi, M. S.; Anal. Methods 2014, 6, 6997.

38. El-Shahawi, M. S.; Bashammakh, A. S.; Al-Sibaai, A. A.; Bahaffi, S. O.; Al-Gohani, E. H.; Electroanalysis 2011, 23, 747.

39. Gamboa, J. C. M.; Cornejo, L.; Acarapi, J.; Squella, J. A.; J. Chil. Chem. Soc. 2013, 4, 2031.

40. Gholivand, M. B.; Pourhossein, A.; Turk. J. Chem. 2011, 35, 839.

41. Baranowska, I.; Kowalski, B.; Polkowska-Motrenko, H.; Samczyński, Z.; Polish J. Environ. Stud. 2015, 24, 1911.

42. Rojas, C.; Arancibia, V.; Gómez, M.; Nagles, E.; Electroanalysis 2013, $25,439$.

43. dos Anjos, V. E.; da C. Machado, E.; Grassi, M. T.; Aquat. Geochem. 2012, 18, 407 .

44. Arab, S.; Alshikh, A.; Nat. Sci. 2010, 8, 292.

45. González, M. J. G.; Renedo, O. D.; Martínez, M. J. A.; Talanta 2007, 71,691 .
46. Nagles, E.; Arancibia, V.; Ríos, R.; Int. J. Eletrochem. Sci. 2012, 7 , 4545.

47. Raj, J.; Raina, A.; Mohineesh; Dogra, T. D.; E3S Web Conf. 2013, 9009 , 3.

48. Rojas, C.; Arancibia, V.; Gómez, M.; Nagles, E.; Sens. Actuators, B 2013, 185,560

49. Sahin, I.; Ertaş, F. N.; Nakiboğlu, N.; Anal. Lett. 2008, 41, 2621.

50. Catrambone, M.; Canepari, S.; Perrino, C.; Web Conf. 2013, 7005, 1.

51. González, A. G.; Herrador, M. Á.; TrAC - Trends Anal. Chem. 2007, 26, 227.

52. Kierszniewska, A.; Krzek, J. A. N.; Acta Pol. Pharm. 2012, 69, 611.

53. Nascimento, P. C.; Schneider, A. B.; Bohrer, D.; de Carvalho, L. M.; Jost, C. L.; J. Braz. Chem. Soc. 2011, 22, 820.

54. de Carvalho, L. M.; Correia, D.; Garcia, S. C.; de Bairros, A. V.; Nascimento, P. C. Do; Bohrer, D.; Forensic Sci. Int. 2010, 202, 75.

55. de Carvalho, L. M.; Spengler, C.; Garmatz, J. C.; do Nascimento, P. C.; Bohrer, D.; Del-Fabro, L.; Radis, G.; Bolli, A. A.; Garcia, S. C.; Moro, A.; da Rosa, M. B.; Quim. Nova 2008, 31, 1336.

56. Attar, T.; Harek, Y.; Larabi, L.; Mediterr. J. Chem. 2014, 2, 691.

57. Alghamdi, A. H.; J. Saudi Chem. Soc. 2010, 14, 1.

58. Melucci, D.; Locatelli, C.; Microchem. J. 2007, 85, 321.

59. Locatelli, C.; Melucci, D.; Food Chem. 2012, 130, 460

60. Suwannasom, P.; Ruangviriyachai, C.; Int. Food Res. J. 2011, 808, 803.

61. Wararattananurak, P.; Chooto, P.; Sherdshoopongse, P.; Chuaynukool, C.; Innuphat, C.; ScienceAsia 2014, 40, 355.

62. Campos, B. K. de; Prazeres, J. P. dos; Torres, Y. R.; Anjos, V. E. dos; Quináia, S. P.; Quim. Nova 2014, 37, 1479.

63. Bento, F. R.; Grassi, M. T.; Sales, A.; Mascaro, L. H.; Int. J. Eletrochem. Sci. 2008, 3, 1523.

64. Trindade, J. M.; Martiniano, L. C.; Gonçalves, V. R. A.; Souza, A. G.; Marques, A. L. B.; Baugis, G. L.; Fonseca, T. C. O.; Song, C.; Zhang, J.; Marques, E. P.; Fuel 2012, 91, 26.

65. Biçer, E.; Arat, C.; J. Chil. Chem. Soc. 2008, 4, 1734.

66. Dadda, A. S.; Teixeira, A. C.; Feltes, P. K.; Campos, M. M.; Leite, C. E.; Moriguchi-Jeckel, C. M.; J. Braz. Chem. Soc. 2014, 25, 1621.

67. Domingos, D.; Ferreira Neta, M. D. L. S.; Massa, A. R. C. G.; Reboucas, M. V.; Teixeira, L. S. G.; J. Braz. Chem. Soc. 2016, 27, 1594

68. de Carvalho, L. M.; Garmatz, J. C.; Spengler, C.; do Nascimento, P. C.; Bohrer, D.; Canabarro, C. M.; Garcia, S. C.; da Rosa, M. B.; J. Braz. Chem. Soc. 2010, 21, 701.

69. de Carvalho, L. M.; do Nascimento, P. C.; Bohrer, D.; Stefanello, R.; Pilau, E. J.; da Rosa, M. B.; Electroanalysis 2008, 20, 776.

70. de Carvalho, J. M.; Leandro, K. C.; Rev. Inst. Adolfo Lutz 2012, 71, 100.

71. Dias, D.; do Nascimento, P. C.; Jost, C. L.; Bohrer, D.; de Carvalho, L. M.; Koschinsky, A.; Electroanalysis 2010, 22, 1066.

72. Erden, S.; Durmus, Z.; Kiliç, E.; Electroanalysis 2011, 23, 1967.

73. Gholivand, M. B.; Sohrabi, A.; Abbasi, S.; Electroanalysis 2007, 19, 1609.

74. Locatelli, C.; J. Sci. Food Agric. 2007, 87, 305

75. Matar, R.; Salami, M.; Int. J. Pharm. Sci. Rev. Res. 2014, 28, 224.

76. Melucci, D.; Locatelli, M.; Locatelli, C.; Food Chem. Toxicol. 2013, 62, 901

77. Mohineesh; Raina, A.; Raj, J.; Dogra, T. D.; E3S Web Conf. 2013, 1, 1

78. Muratt, D. T.; M. de Carvalho, L.; Viana, C.; C. do Nascimento, P.; Reis, G.; R. Dal Molin, T.; Grassmann, C.; Smidt, G.; Koschinsky, A.; Electroanalysis 2015, 27, 1625.

79. Nosal-Wiercińska, A.; Dalmata, G.; Monatsh. Chem. 2009, 140, 1421.

80. Nascimento, P. C.; Del-Fabro, L. D.; Bohrer, D.; De Carvalho, L. M.; Rosa, M. B.; Noremberg, S. M.; Electroanalysis 2008, 20, 1078.

81. do Nascimento, P. C.; Jost, C. L.; de Carvalho, L. M.; Bohrer, D.; Koschinsky, A.; Anal. Chim. Acta 2009, 648, 162.

82. Nascimento, D. S.; Insausti, M.; Band, B. S. F.; Lemos, S. G.; Fuel 2014, 137, 172 
83. Opoka, W.; Adamek, D.; Plonka, M.; Reczynski, W.; Bas, B.; Drozdowicz, D.; Jagielski, P.; Sliwowski, Z.; Adamski, P.; Brzozowski, T.; J. Physiol. Pharmacol. 2010, 61, 581.

84. Opydo, J.; Proc. ECOpole 2008, 2, 363.

85. Rojas-Romo, C.; Arancibia, V.; Moreno-Da Costa, D.; Tapia, R. A.; Sens. Actuators, B 2016, 224, 772.

86. Shahbazi, Y.; Ahmadi, F.; Fakhari, F.; Food Chem. 2016, 192, 1060.

87. Scheffer, E. W. O.; Grassi, M. T.; Quim. Nova 2010, 33, 1254.

88. Sobhanardakani, S.; Tayebi, L.; Farmany, A.; World Appl. Sci. J. 2011, 14, 1453.

89. Sodré, F. F.; Schnitzler, D. C.; Scheffer, E. W. O.; Grassi, M. T.; Aquat. Geochem. 2012, 18, 389.

90. Yilmaz, S.; Öztürk, B.; Özdemir, D.; Eroğlu, A. E.; Ertaş, F. N.; Turk. J. Chem. 2013, 37, 316.

91. Zarei, K.; Atabati, M.; Karami, M.; Anal. Chim. Acta 2009, 649, 62.

92. Nunes, C. N.; Pauluk, L. E.; dos Anjos, V. E.; Lopes, M. C.; Quináia, S. P.; Anal. Bioanal. Chem. 2015, 407, 6171.

93. Nunes, C. N.; Pauluk, L. E.; Felsner, M. L.; Egéa, V.; Quináia, S. P.; J. Anal. Methods Chem. 2016, 2016, 1.

94. dos Santos, L. B. O.; Abate, G.; Masini, J. C.; J. Braz. Chem. Soc. 2006, 17,36 .

95. Miranda, L.; Galli, A.; Quináia, S. P.; Rev. Virtual Quim. 2014, 6, 416.

96. Ramadan, A. A.; Mandil, H.; Ghazal, N.; Innovare Acad. Sci. 2015, 7, 300.

97. El-Desoky, H. S.; Ghoneim, M. M.; Abdel-Galeil, M. M.; J. Braz. Chem. Soc. 2010, 21, 669 .

98. Al-Ghamdi, A. F.; J. Saudi Chem. Soc. 2009, 13, 235.

99. Beltagi, A. M.; El-Attar, M. A.; Ghoneim, E. M.; Cent. Eur. J. Chem. 2007, 5, 835 .

100. Jain, R.; Sharma, R.; J. Pharm. Anal. 2012, 2, 447.

101. Samiec, P.; Navrátilová, Z.; Fischer, J.; Monatsh. Chem. 2016, 147, 127.

102. Jain, R.; Radhapyari, K.; Jadon, N.; J. Electrochem. Soc. 2007, 154, F199.

103. Hoang, V. D.; Yen, N. T.; Trop. J. Pharm. Res. 2013, 12, 783.

104. Ghoneim, M. M.; Abdel-Azzem, M. K.; El-Desoky, H. S.; Ghoneim, A. M.; Khattab, A. E.; J. Braz. Chem. Soc. 2014, 25, 1407.

105. Taşdemir, I. H.; J. Food Drug Anal. 2014, 22, 527.

106. Guziejewski, D.; Brycht, M.; Nosal-Wiercinska, A.; Smarzewska, S.; Ciesielski, W.; Skrzypek, S.; J. Environ. Sci. Health, Part B 2014, 49, 550 .

107. Erdoğdu, G.; J. Anal. Chem. 2008, 63, 762.

108. dos Santos, S. C.; Galli, A.; Felsner, M. L.; Justi, K. C.; Rev. Virtual Quim. 2014, 6, 866.

109. Kumar, K. N. S.; Sreedhar, N. Y.; Indian J. Adv. Chem. Sci. 2012, 1, 33. 110. Pelit, F. O.; Ertas, H.; Ertaş, F. N.; J. Appl. Electrochem. 2011, 41, 1279.

111. El-Shahawi, M. S.; Hamza, A.; Bashammakh, A. S.; Al-Sibaai, A. A.; Al-Saggaf, W. T.; Electroanalysis 2011, 23, 1175.

112. Qiu, P.; Tian, X. C.; Asian J. Chem. 2013, 25, 4457.

113. Wyantuti, S.; Tjokronegoro, R.; Rochani, S.; Proceeding Int. Semin. Chem. 2008, 2008, 374.

114. Galli, A.; De Souza, D.; Machado, S. A. S.; Microchem. J. 2011, 98 , 135.

115. Večerková, R.; Hernychová, L.; Dobeš, P.; Vrba, J.; Josypčuk, B.; Bartošík, M.; Vacek, J.; Anal. Chim. Acta 2014, 830, 23.

116. Kumar, K. N. S.; Sreedhar, N. Y.; Indian J. Adv. Chem. Sci. 2016, 4, 484.

117. Zainudin, N. S.; Yaacob, M. H.; Muslim, N. Z. M.; Malaysian J. Anal. Sci. 2016, 20, 1254.

118. Babu, T. R.; Sivasankar, K.; Sujana, P.; Environ. Sci. 2012, 7, 5.

119. Carvalho, J. M.; Silva, A. R. da; Cunha, A. L. M. C. da; Aucélio, R. Q.; Alberti, A. L. M.; Leandro, K. C.; Quim. Nova 2012, 35, 988.

120. da Silva, J. G.; e Silva, M. R. L.; de Oliveira, A. C.; de Souza, J. R.; Vaz, C. M. P.; de Castro, C. S. P.; J. Food Compos. Anal. 2012, 25, 1.

121. Ensaifi, A. A.; Khayamian, T.; Taei, M.; Talanta 2009, 78, 942.
122. Al-Ghamdi, A. F.; Hefnawy, M. M.; Al-Majed, A. A.; Belal, F. F.; Chem. Cent. J. 2012, 6, 362.

123. Černocká, H.; Ostatná, V.; Paleček, E.; Electrochem. Commun. 2015, 61, 114.

124. de Carvalho, L. M.; do Nascimento, P. C.; Bohrer, D.; Correia, D.; de Bairros, A. V.; Pomblumc, V. J.; Pomblum, S. G.; J. Braz. Chem. Soc. 2007, 18, 789.

125. Adam, V.; Krizkova, S.; Zitka, O.; Trnkova, L.; Petrlova, J.; Beklova, M.; Kizek, R.; Electroanalysis 2007, 19, 339.

126. Garbellini, G. S.; Pedrosa, V. A.; Salazar-Banda, G. R.; Avaca, L. A.; Quim. Nova 2007, 30, 2025.

127. Taşdemir, I. H.; Arabian J. Chem. 2016, 9, 86.

128. Hoang, V. D.; Huyen, D. T.; Phuc, P. H.; J. Anal. Chem. 2013, 12, 1.

129. Jain, R.; Dwivedi, A.; Mishra, R.; Langmuir 2009, 25, 10364.

130. Al-Omar, M. A.; Al-ghamdi, A. F.; Al-ghamdi, A. H.; Saudi Pharm. J. 2008, 16, 231.

131. Lakshmi, D.; Sharma, P. S.; Prasad, B. B.; Biosens. Bioelectron. 2007, 22, 3302

132. El-Shal, M. A.; J. Pharm. Res. 2012, 5, 280.

133. Guziejewski, D.; Skrzypek, S.; Ciesielski, W.; Environ. Monit. Assess. 2012, 184, 6575.

134. Nascimento, P. C.; Hilgemann, M.; Guterres, M. V.; de Carvalho, L. M.; Bohrer, D.; Chemom. Intell. Lab. Syst. 2007, 89, 97.

135. Sreedhar, N. Y.; Sreenivasulu, A.; Sunil Kumar, M.; Nagaraju, M.; Int. J. PharmTech Res. 2012, 4, 1303.

136. Jain, R.; Yadav, R. K.; Dwivedi, A.; Colloids Surfaces A Physicochem. Eng. Asp. 2010, 359, 25.

137. Alghamdi, A. F.; Kooli, F.; J. Mater. Environ. Sci. 2013, 4, 762.

138. Habib, I. H. I.; Rizk, M. S.; El-Alamin, M. M. A.; Imam, G. S.; Port. Electrochim. Acta 2016, 34, 343.

139. Pawlak, M. K.; Ecol. Chem. Eng. S 2008, 15, 263.

140. Eş, Z.; Taşdemir, I. H.; Turk. J. Chem. 2015, 39, 54.

141. El-desoky, H. S.; J. Braz. Chem. Soc. 2009, 20, 1790.

142. Reddy, C. N.; Prasad, P. R.; Sreedhar, N. Y.; Int. J. Pharm. Pharm. Sci. 2011, 3, 141.

143. Ribeiro, F. W. P.; Mendonça, G. L. F.; Soares, J. E. S.; Freire, V. N.; de Souza, D.; Casciano, P. N. S.; de Lima-Neto, P.; Correia, A. N.; Electrochim. Acta 2014, 137, 564.

144. Temerk, Y. M.; Ibrahim, M. S.; Kotb, M.; J. Braz. Chem. Soc. 2011, 22, 2056.

145. El-Desoky, H. S.; Ghoneim, M. M.; Habazy, A. D.; J. Braz. Chem. Soc. 2011, 22, 239.

146. Ghani, N. T. A.; El-Ries, M. A.; El-Shall, M. A.; Anal. Sci. 2007, 23, 1053.

147. Habib, I. H. I.; Weshahy, S. A.; Toubar, S.; Port. Electrochim. Acta 2008, 4,315 .

148. Erdoğdu, G.; Titretir, S.; J. Anal. Chem. 2007, 62, 777.

149. Mirceski, V.; Guziejewski, D.; Skrzypek, S.; Ciesielski, W.; Croat. Chem. Acta 2010, 83, 121.

150. Miranda, L.; Felsner, M. L.; Torres, Y. R.; Hoss, I.; Galli, A.; Quináia, S. P.; Quim. Nova 2015, 38, 419.

151. Alghamdi, A. F.; Port. Electrochim. Acta 2014, 32, 51.

152. Thuong, N. T. K.; Huyen, T. C.; J. Chem. 2010, 48, 604.

153.Gupta, V. K.; Jain, R.; Antonijevic, M. M.; Khani, H.; Siddiqui, M. N.; Dwivedi, A.; Mishra, R.; Agarwal, S.; Int. J. Electrochem. Sci. 2011, 6, 37.

154. Guziejewski, D.; Nosal-wierci, A.; Skrzypek, S.; Ciesielski, W.; Smarzewska, S.; J. Chem. 2016, 2016, 1.

155. Biçer, E.; Çetinkaya, P.; J. Chil. Chem. Soc. 2009, 1, 46.

156. Öztürk, F.; Küçükkolbasi, S.; Kaçar, C.; Kiliç, E.; J. Braz. Chem. Soc. 2014, 25, 920.

157. Sreedhar, N. Y.; Sreenivasulu, A.; Sunil Kumar, M.; Nagaraju, M.; Int. J. Pharm. Sci. Res. 2012, 3, 2320. 
158. Pushpalatha, M.; Balaji, K.; Reddy, C. S.; Int. J. Chem. Sci. Technol. 2011, $1,171$.

159. Alghamdi, A. F.; J. Mol. Liq. 2016, 218, 186.

160. Dar, R. A.; Brahman, P. K.; Tiwari, S.; Pitre, K. S.; Colloids Surf., B 2012, 98, 72.

161. Paleček, E.; Ř́mánková, L.; Electrochem. Commun. 2014, 44, 59.

162. Adnan, L. A.; Yusoff, A. R. M.; Hadibarata, T.; Khudhair, A. B.; Water. Air. Soil Pollut. 2014, 225, 2119.

163. Mandil, H.; Sakur, A. A.; Alulu, S.; Int. J. Pharm. Pharm. Sci. 2013, 5, 502.

164. Guida, M.; Maria, M. S.; Salvatore, F.; Biochem. Physiol.: Open Access $\mathbf{2 0 1 5}, 4,1$.

165. Al-Ghamdi, A. F.; Hefnawy, M. M.; Arabian J. Chem. 2012, 5, 383.

166. Cvrkovic-Karloci, Ž.; Krznaric, D.; Šeruga, M.; Cosovic, B.; Int. J. Electrochem. 2011, 2011, 1.

167. Frka, S.; Dautović, J.; Kozarac, Z.; Ćosović, B.; Hoffer, A.; Kiss, G.; Tellus B 2012, 64, 1 .
168. Gómez, M.; Arancibia, V.; Aliaga, M.; Núnez, C.; Rojas-Romo, C.; Food Chem. 2016, 212, 807.

169. Majnooni, M. B.; Gholivand, M. B.; Khodadadian, M.; Afnanzade, N.; Bahrami, G.; J. Rep. Pharm. Sci. 2012, 1, 34.

170. Gómez, M.; Arancibia, V.; Rojas, C.; Nagles, E.; Int. J. Electrochem. Sci. 2012, 7, 7493.

171. Al-Ghamdi, A. F.; Am. J. Anal. Chem. 2011, 2, 174.

172. Kaçar, C.; Durmus, Z.; Kiliç, E.; Asian J. Chem. 2014, 26, 1931.

173. Bandzuchová, L.; Selesovská, R.; Navrátil, T.; Chylková, J.; Electrochim. Acta 2013, 113, 1.

174. Habib, I. H. I.; Weshahy, S. A.; Toubar, S.; El-Alamin, M. M. A.; Pharmazie 2008, 63, 337.

175. Leandro, K. C.; Moreira, J. C.; Farias, P. A. M.; J. Pharm. 2013, 2013, 1.

176. Reddy, C. N.; Prasad, P. R.; Sreedhar, N. Y.; J. Anal. Methods Chem. 2013, 2013, 1.

177. Reddy, C. N.; Chandrasekhar, G.; Sreedhar, N. Y.; Anal. Pharm. Biomed. Sci. 2013, 2, 27. 